MATHEMATICS OF COMPUTATION

Volume 80, Number 274, April 2011, Pages 821-846

S $0025-5718(2010) 02402-0$

Article electronically published on September 17, 2010

\title{
OPERATOR SPLITTING FOR THE KdV EQUATION
}

\author{
HELGE HOLDEN, KENNETH H. KARLSEN, NILS HENRIK RISEBRO, \\ AND TERENCE TAO
}

\begin{abstract}
We provide a new analytical approach to operator splitting for equations of the type $u_{t}=A u+B(u)$, where $A$ is a linear operator and $B$ is quadratic. A particular example is the Korteweg-de Vries (KdV) equation $u_{t}-u u_{x}+u_{x x x}=0$. We show that the Godunov and Strang splitting methods converge with the expected rates if the initial data are sufficiently regular.
\end{abstract}

\section{INTRODUCTION}

The ubiquitous Korteweg-de Vries (KdV) equation

$$
u_{t}-u u_{x}+u_{x x x}=0
$$

offers the perfect blend of the simplest nonlinear convective term $u u_{x}$ and the simplest dispersive term $u_{x x x}$ in that the well-known smooth soliton solutions of the KdV equation interact in an almost linear fashion apart from a phase shift. Furthermore, the KdV equation is completely integrable with an infinite family of conserved quantities. This is a result of a subtle interaction between the Burgers term $u u_{x}$ and the Airy term $u_{x x x}$, as it is a well-known fact that the nonlinear Burgers equation $u_{t}-u u_{x}=0$ generically develops shocks in finite time while the linear Airy equation $u_{t}+u_{x x x}=0$ preserves all Sobolev norms.

The initial value problem for the KdV equation with $\left.u\right|_{t=0}=u_{0} \in H^{s}$, either on the whole real line or in the periodic case, has been extensively studied. An incomplete list of references is [2, 5]. For further discussions we refer to [8, 6].

The method of operator splitting, also called the fractional steps method, remains a very popular method both for analysis and numerical computations of partial differential equations. Instead of including a long list of references to relevant works, we entrust the reader instead with 4 and the overview of the field given therein. However, we refer to 7 for rigorous analysis of the splitting method applied to the Schrödinger-Poisson and the cubic nonlinear Schrödinger equations.

Formally operator splitting can be explained as follows. Let $u(t)=\Phi_{C}(t) u_{0}=$ $\Phi_{C}\left(t ; u_{0}\right) \in X$, where $X$ is some normed space, denote the solution of a given

Received by the editor June 6, 2009 and, in revised form, December 9, 2009.

2010 Mathematics Subject Classification. Primary 35Q53; Secondary 65M12, 65M15.

Key words and phrases. KdV equation, operator splitting.

Supported in part by the Research Council of Norway. This paper was written as part of the international research program on Nonlinear Partial Differential Equations at the Centre for Advanced Study at the Norwegian Academy of Science and Letters in Oslo during the academic year 2008-09. The fourth author is supported by a grant from the MacArthur Foundation, the NSF Waterman award, and NSF grant DMS-0649473.

(C)2010 American Mathematical Society Reverts to public domain 28 years from publication 
differential equation:

$$
u_{t}=C(u), \quad t \in[0, T],\left.\quad u\right|_{t=0}=u_{0} .
$$

Here $C$ will typically be a differential operator in the spatial variable. Assuming that we can write $C=A+B$ in a natural way, the idea of operator splitting is that

$$
u\left(t_{n}\right) \approx\left(\Phi_{B}(\Delta t) \Phi_{A}(\Delta t)\right)^{n} u_{0}, \quad t_{n}=n \Delta t,
$$

where $\Delta t \ll 1$. In the case of linear ordinary differential equations, this goes back to Sophus Lie. One of the reasons for its popularity is that operator splitting allows for a separate treatment of the equations $u_{t}=A(u)$ and $u_{t}=B(u)$; in particular this applies to the use of dedicated special numerical techniques for each of the equations; again we refer to 4 for a long list of examples and relevant references.

In the context of the KdV equation, the first use of operator splitting was reported in the brief paper by Tappert [9], where it was applied as a numerical method. Apparently the first rigorous results appeared in [3] where a Lax-Wendroff result was proved: If operator splitting converges to some limit function, then the limit function is a weak solution of the $\mathrm{KdV}$ equation. In addition a systematic study of operator splitting as a numerical method was undertaken for the KdV equation. More extensive rigorous results, specifically the convergence of the splitting approximations in a suitable functional space, were hampered by the apparent incompatibility between the Burgers equation and the Airy equation.

In the present paper we offer a new analytical approach to operator splitting for the KdV equation that will lead to rigorous convergence results (error estimates). Compared to earlier attempts, two new ingredients enter the present approach. First of all we actively use that the solution of the KdV equation remains bounded in a Sobolev space; that is, if $u_{0} \in H^{s}(\mathbb{R})$, then $\|u(t)\|_{H^{s}(\mathbb{R})}$ remains bounded for $t \in[0, T]$. This together with a bootstrap argument is used to secure the existence of a uniform choice of time step $\Delta t$ that prevents the solution from any "Burgers" step from blowing up. Indeed the main problem in this approach is that the Airy equation produces small oscillatory waves that, when used as initial data for the Burgers equation, produce shocks. Secondly, since the splitting approximations are merely defined at the discrete times $t=t_{n}$, to facilitate the convergence analysis we introduce an extension which is defined for all $t \in[0, T]$. Concretely, we introduce an extension $v$ which depends on an additional time variable $\tau \in[0, T]$, i.e., $v=v(t, \tau)$, and let the evolution corresponding to each time variable be governed by one of the split operators in such a way that at each time level $t=t_{n}$ the extension $v\left(t_{n}, t_{n}\right)$ coincides with the regular splitting approximation. This extension approach is different from the conventional one, where one lets "time run twice as fast" in each of the subintervals $\left[t_{n}, t_{n}+\Delta t / 2\right]$ and $\left[t_{n}+\Delta t / 2, t_{n+1}\right]$ (cf. the discussion and references in [4]).

Formally the operator splitting (11), called sequential or Godunov splitting, yields a first-order approximation in $\Delta t$, that is,

$$
\left\|u\left(t_{n}\right)-\left(\Phi_{A}(\Delta t) \Phi_{B}(\Delta t)\right)^{n} u_{0}\right\|=\mathcal{O}(\Delta t), \quad t_{n} \rightarrow t, \Delta t \rightarrow 0,
$$

in an appropriate norm. We show that this holds rigorously for the KdV equation. More precisely, we prove in Theorem 2.4 that for $u_{0} \in H^{s}(\mathbb{R})$ with $s \geq 5$ we have that for $\Delta t$ sufficiently small,

$$
\|v(t, t)-u(t)\|_{H^{s-3}(\mathbb{R})} \leq K \Delta t, \quad t \in[0, T],
$$


where $K$ depends on $s, T$ and $u_{0}$ only. Here $v(t, t)$ denotes the splitting approximation in our approach evaluated on the "diagonal" $(t, \tau)=(t, t)$.

To obtain second-order convergence, it is common to apply the Strang splitting formula, thus formally

$$
\left\|u\left(t_{n}\right)-\left(\left(\Phi_{B}(\Delta t / 2) \Phi_{A}(\Delta t / 2)\right)\left(\Phi_{A}(\Delta t / 2) \Phi_{B}(\Delta t / 2)\right)\right)^{n} u_{0}\right\|=\mathcal{O}\left(\Delta t^{2}\right),
$$

where $t_{n} \rightarrow t$ as $\Delta t \rightarrow 0$. Here we show rigorously this result for the KdV equation. Indeed, in Theorem 3.5. we prove that if $u_{0} \in H^{s}$ for some $s \geq 17$, then for $\Delta t$ sufficiently small,

$$
\|v(t, t)-u(t)\|_{H^{s-9}} \leq K \Delta t^{2}, \quad t \in[0, T],
$$

where the constant $K$ depends on $u_{0}, s$ and $T$ only. Again $v(t, t)$ denotes the operator splitting approximation in our approach. Observe that we have to increase the regularity of the initial data, and hence of the solution, in order to get increased accuracy of operator splitting. Note also that with this type of operator splitting, our error estimates are in a much weaker norm than the assumptions.

It is clear that the present approach applies to several other equations, and this is currently being investigated. Furthermore, for applications to numerical analysis, one would need to replace the exact solution operators $\Phi_{A}$ and $\Phi_{B}$ by numerical approximations, say $\Phi_{A}^{\delta}$ and $\Phi_{B}^{\delta}$, and study their behavior in the limit as $\delta \rightarrow 0$, and also to replace the time derivatives by discrete differences. Again this will be studied separately.

The paper is organized as follows. We start by presenting the Godunov operator splitting method for abstract operators. Next we apply this approach to nonlinear ordinary differential equations where the procedure is fairly transparent, before we discuss Godunov splitting for the KdV equation. Subsequently we present the Strang splitting for abstract operators. Finally we apply this procedure to the KdV equation.

\section{Operator Splitting}

We first present a formal calculation motivating the rigorous analysis that will follow. Consider an abstract differential equation

$$
u_{t}=C(u), \quad t \in[0, T],\left.\quad u\right|_{t=0}=u_{0}
$$

for some fixed positive time $T$, where $C$ typically will be a differential operator in the spatial variable. We assume that $u_{0}$ and $u$ are in some Hilbert space $X$, and we write the solution as

$$
u(t)=\Phi_{C}\left(t ; u_{0}\right) .
$$

Formally, expanding the solution in a Taylor series we find

$$
u(t)=u_{0}+t u_{t}(0)+\mathcal{O}\left(t^{2}\right)=u_{0}+t C\left(u_{0}\right)+\mathcal{O}\left(t^{2}\right),
$$

where we have used the equation (2). Assume that one can write

$$
C=A+B
$$

in some natural way. Operator splitting (of the Godunov type) then works as follows. Instead of solving the problem with $C$ directly, one alternately solves for small time steps the equations $u_{t}=A(u)$ and $u_{t}=B(u)$. Making the time steps finer and finer, the approximation will presumably converge to the solution of the original equation (2). 
More precisely, fix a positive time step $\Delta t$, let $t_{n}=n \Delta t, n \in \mathbb{N}_{0}$, and define a family $\left\{u_{\Delta t}\left(t_{n}\right)\right\}_{n}$ of functions

$$
\begin{aligned}
u_{\Delta t}\left(t_{n+1}\right) & =\Phi_{A}\left(\Delta t ; \Phi_{B}\left(\Delta t ; u_{\Delta t}\left(t_{n}\right)\right)\right)=\Phi_{A}(\Delta t) \circ \Phi_{B}(\Delta t) u_{\Delta t}\left(t_{n}\right), \quad n \in \mathbb{N}_{0}, \\
u_{\Delta t}(0) & =u_{0} .
\end{aligned}
$$

The traditional method of extending the solution to any $t \in[0, T]$ has been to let "time run twice as fast" in each of the subintervals $\left[t_{n}, t_{n+1 / 2}\right]$ and $\left[t_{n+1 / 2}, t_{n+1}\right]$, where $t_{n+1 / 2}=t_{n}+\Delta t / 2$. Thus obtaining

$$
u_{\Delta t}(t)= \begin{cases}\Phi_{B}\left(2\left(t-t_{n}\right) ; u_{\Delta t}\left(t_{n}\right)\right) & \text { for } t \in\left[t_{n}, t_{n+1 / 2}\right], \\ \Phi_{A}\left(2\left(t-t_{n+1 / 2}\right) ; u_{\Delta t}\left(t_{n+1 / 2}\right)\right) & \text { for } t \in\left[t_{n+1 / 2}, t_{n+1}\right] .\end{cases}
$$

(In the present approach we will use a different extension to all times $t$.)

Formally one can show that

$$
\left\|u_{\Delta t}\left(t_{n}\right)-u\left(t_{n}\right)\right\| \leq \mathcal{O}(\Delta t) \text { as } \Delta t \rightarrow 0 \text { and } t_{n} \rightarrow t
$$

in some norm.

The convergence can be improved to second order by using the Strang splitting formula. To this end we let the approximation (this time denoted $v_{\Delta t}$ to distinguish from the previous approximation) read

$$
\begin{aligned}
v_{\Delta t}\left(t_{n+1}\right)= & \Phi_{B}\left(\Delta t / 2 ; \Phi_{A}\left(\Delta t ; \Phi_{B}\left(\Delta t / 2 ; v_{\Delta t}\left(t_{n}\right)\right)\right)\right) \\
= & \Phi_{B}(\Delta t / 2) \circ \Phi_{A}(\Delta t) \circ \Phi_{B}(\Delta t / 2) v_{\Delta t}\left(t_{n}\right) \\
= & \left(\Phi_{B}(\Delta t / 2) \circ \Phi_{A}(\Delta t / 2)\right) \\
& \quad \circ\left(\Phi_{A}(\Delta t / 2) \circ \Phi_{B}(\Delta t / 2)\right) v_{\Delta t}\left(t_{n}\right), \quad n \in \mathbb{N}_{0}, \\
v_{\Delta t}(0)= & u_{0} .
\end{aligned}
$$

Formally one now has

$$
\left\|v_{\Delta t}\left(t_{n}\right)-u\left(t_{n}\right)\right\| \leq \mathcal{O}\left(\Delta t^{2}\right) \text { as } \Delta t \rightarrow 0 \text { and } t_{n} \rightarrow t .
$$

To show that the operator splitting solutions are well defined, we shall later make use of the following bootstrap lemma, taken from [8, Prop. 1.21].

Lemma 2.1. Let $t \in[0, T]$. Consider a continuous function $\phi:[0, T] \rightarrow[0, \infty)$. If there exists a positive constant $\alpha$ such that

(a) $\phi(0) \leq \alpha$,

(b) for any $t$ such that $\phi(t) \leq \alpha$, we can show that $\phi(t) \leq \alpha / 2$, then $\phi(t) \leq \alpha / 2$ for all $t \in[0, T]$.

2.1. Doubling the time variable. We shall formulate the operator splitting solution by introducing two time variables, and define a function $v=v(t, \tau)$ for $(t, \tau)$ in the set

$$
\Omega_{\Delta t}=\bigcup_{n=0}^{\lfloor T / \Delta t\rfloor}\left[t_{n}, t_{n+1}\right] \times\left[t_{n}, t_{n+1}\right]
$$

by requiring that

$$
\begin{aligned}
v(0,0) & =u_{0} \\
v_{t}\left(t, t_{n}\right) & =B\left(v\left(t, t_{n}\right)\right), \quad t \in\left(t_{n}, t_{n+1}\right], \\
v_{\tau}(t, \tau) & =A(v(t, \tau)), \quad(t, \tau) \in\left[t_{n}, t_{n+1}\right] \times\left(t_{n}, t_{n+1}\right],
\end{aligned}
$$




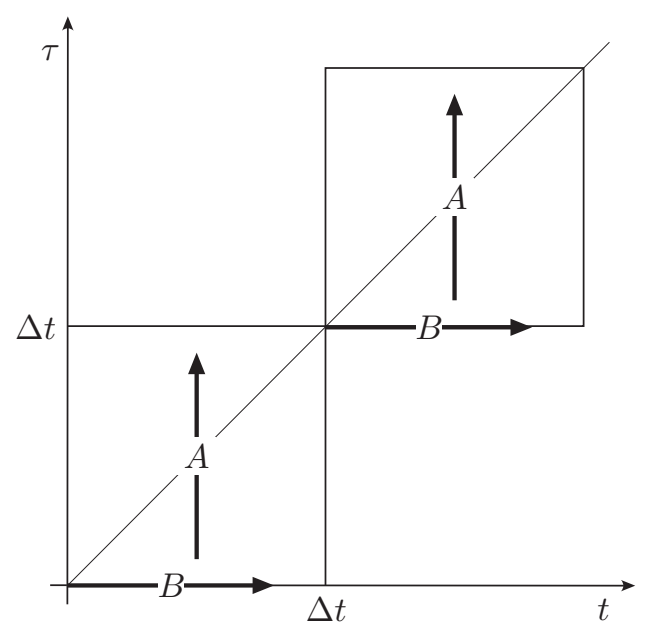

Figure 1. Schematic view of the Godunov splitting and the definition of $v(t, \tau)$; cf. equation (4).

where $n=0, \ldots,\lfloor T / \Delta t\rfloor$. Observe that

$$
u_{\Delta t}\left(t_{n}\right)=v\left(t_{n}, t_{n}\right), \quad n=0, \ldots,\lfloor T / \Delta t\rfloor,
$$

where $u_{\Delta t}$ is given by (3). The specific extension $v$ of $\left\{u_{\Delta t}\left(t_{n}\right)\right\}_{n}$ to $[0, T]$ will serve as an important technical tool to be utilized in the analysis.

The exact solution of (2) is still denoted by $u$. Introduce the error function

$$
w(t)=v(t, t)-u(t) .
$$

The aim is to show that

$$
\|w(t)\| \leq \mathcal{O}(\Delta t), \quad \Delta t \rightarrow 0, \quad t \in[0, T]
$$

in an appropriate norm.

We introduce second-order Taylor expansions of the operators $A$ and $B$ (see 1, p. 29]),

$$
\begin{aligned}
& A(f+g)=A(f)+d A(f)[g]+\int_{0}^{1}(1-\alpha) d^{(2)} A(f+\alpha g)[g]^{2} d \alpha, \\
& B(f+g)=B(f)+d B(f)[g]+\int_{0}^{1}(1-\alpha) d^{(2)} B(f+\alpha g)[g]^{2} d \alpha .
\end{aligned}
$$

Thus we find

$$
\begin{aligned}
w_{t}-d A(u)[w]-d B(u)[w]= & v_{t}+v_{\tau}-u_{t}-d A(u)[w]-d B(u)[w] \\
= & v_{t}+A(v)-(A+B)(u)-d A(u)[w]-d B(u)[w] \\
= & v_{t}-B(v)+(A(v)-A(u)-d A(u)[w]) \\
& \quad+(B(v)-B(u)-d B(u)[w]) \\
= & F(t)+\int_{0}^{1}(1-\alpha) d^{(2)} A(u+\alpha w)[w]^{2} d \alpha \\
& +\int_{0}^{1}(1-\alpha) d^{(2)} B(u+\alpha w)[w]^{2} d \alpha,
\end{aligned}
$$


where we have introduced a forcing term $F(t)=F(t, t)$ defined as

$$
F(t, \tau)=v_{t}(t, \tau)-B(v(t, \tau))
$$

We can rewrite (5) as follows:

$$
w_{t}-d C(u)[w]=F(t)+\int_{0}^{1}(1-\alpha) d^{(2)} C(u+\alpha w)[w]^{2} d \alpha .
$$

The forcing term satisfies the following time development:

$$
\begin{aligned}
F_{\tau}-d A(v)[F] & =v_{t \tau}-B(v)_{\tau}-d A(v)\left[v_{t}-B(v)\right] \\
& =A(v)_{t}-d B(v)\left[v_{\tau}\right]-d A(v)\left[v_{t}\right]+d A(v)[B(v)] \\
& =d A(v)\left[v_{t}\right]-d B(v)[A(v)]-d A(v)\left[v_{t}\right]+d A(v)[B(v)] \\
& =[A, B](v, v),
\end{aligned}
$$

where we have defined the commutator

$$
[A, B](f, g)=d A(f)[B(g)]-d B(f)[A(g)] .
$$

For simplicity we will subsequently be writing $[A, B](v)$ rather than $[A, B](v, v)$.

2.2. Ordinary differential equations. As a warm-up we consider the ordinary differential equation

$$
u_{t}=C(u), \quad t>0, \quad u(0)=u_{0} \in \mathbb{R}^{n} .
$$

To simplify the presentation we assume that $C$ is a quadratic function, i.e., that $C^{\prime \prime \prime}=d^{3} C=0$ or $d^{2} C[f, g]$ is constant. This means that the integral in (7) reduces to a constant. Furthermore, we assume that (9) is such that there is a unique solution $u(t)$ such that $|u(t)| \leq K_{u_{0}, T}$ for $t \in[0, T]$. Furthermore, we will assume that the operators $A$ and $B$ are two times continuously differentiable and

$$
A^{\prime}, A^{\prime \prime} \in L^{\infty}, \quad A(0)=0, \quad|B(u)| \leq K|u|^{2}, \quad\left|B^{\prime}(u)\right| \leq K|u| .
$$

Throughout this paper we use the convention that for a quantity $\alpha, K_{\alpha}$ denotes a constant depending on $\alpha$ (and perhaps other things). We use this notation to highlight the dependence on $\alpha$. The actual value of $K_{\alpha}$ may be different at each occurrence.

Now let $\alpha$ be a positive constant (its precise value will be fixed later). To start the bootstrap argument we assume

$$
|v(t, \tau)| \leq \alpha, \quad(t, \tau) \in \Omega_{\Delta t} .
$$

In this example, since $C$ is quadratic, $d^{2} C=\kappa$ for some constant symmetric matrix $\kappa$, and $w$ satisfies

$$
w_{t}+C^{\prime}(u) w=F+w^{T} \frac{\kappa}{2} w, \quad t>0, \quad w(0)=0 .
$$

Furthermore, $F$ satisfies

$$
F_{\tau}-A^{\prime}(v) F=[A, B](v), \quad(t, \tau) \in\left[t_{n}, t_{n+1}\right] \times\left(t_{n}, t_{n+1}\right],
$$

and $F\left(t, t_{n}\right)=0$, for each $n$. This means that

$$
\frac{\partial}{\partial \tau}|F| \leq K|F|+K \alpha^{2}
$$

Hence, Gronwall's inequality implies that

$$
|F|(t, \tau) \leq K_{\alpha} \Delta t
$$


where $K_{\alpha}$ is a constant depending on the assumed bound on $v$ in (11). In view of this bound and (12),

$$
\frac{d}{d t}|w| \leq K_{\alpha}|w|+K_{\alpha} \Delta t, \quad w(0)=0 .
$$

Gronwall's inequality gives that

$$
|w(t)| \leq e^{K_{\alpha} t} t K_{\alpha} \Delta t \leq K_{\alpha} \Delta t .
$$

Trivially we have

$$
|v(t, t)-v(t, \tau)| \leq \int_{\min \{t, \tau\}}^{\max \{t, \tau\}}|A(v(t, s))| d s \leq K_{\alpha} \Delta t,
$$

for $(t, \tau) \in\left[t_{n}, t_{n+1}\right] \times\left(t_{n}, t_{n+1}\right]$ for any $n$. Then we can conclude that

$$
|v(t, \tau)| \leq|u(t)|+|w(t)|+|v(t, t)-v(t, \tau)| \leq K+K_{\alpha} \Delta t .
$$

Now we are in a position to choose $\alpha$ so that $K \leq \alpha / 4$; this determines $K_{\alpha}$. Next choose $\Delta t$ so small that $K_{\alpha} \Delta t \leq \alpha / 4$. Then

$$
|v(t, \tau)| \leq \alpha / 2, \quad(t, \tau) \in \Omega_{\Delta t} .
$$

Hence, by the bootstrap lemma, $|v(t, t)| \leq \alpha / 2$ for all $t$. Consequently,

$$
|v(t, t)-u(t)| \leq K_{\alpha / 2} \Delta t
$$

i.e., the operator splitting is, as expected, first-order accurate.

Remark 2.2. An interesting example is the logistic equation $u^{\prime}=u(u-1)$, where we can write $A(u)=-u$ and $B(u)=u^{2}$. Exact solutions are available for all operators involved, specifically

$$
\Phi_{C}(t) u_{0}=\frac{u_{0}}{u_{0}+e^{t}\left(1-u_{0}\right)}, \quad \Phi_{A}(t) u_{0}=u_{0} e^{-t}, \quad \Phi_{B}(t) u_{0}=\frac{u_{0}}{1-u_{0} t} .
$$

Let $u_{0} \in(0,1)$. Then there is no blowup in the full equation, but blowup for the equation $u_{t}=b(u)$ at $t^{*}=1 / u_{0}$. The function $v$ reads in our case

$$
v(t, \tau)=\frac{v\left(t_{n}, t_{n}\right) e^{-\left(\tau-t_{n}\right)}}{1-v\left(t_{n}, t_{n}\right)\left(t-t_{n}\right)}, \quad t, \tau \in\left[t_{n}, t_{n+1}\right],
$$

where

$$
v\left(t_{n}, t_{n}\right)=\frac{u_{0}\left(1-e^{-\Delta t}\right)}{\left(1-e^{-\Delta t}\right) e^{t_{n}}+u_{0} \Delta t\left(1-e^{t_{n}}\right)} .
$$

The quantity $v\left(t_{n}, t_{n}\right)$ is well-defined on $[0, T]$ if one chooses $\Delta t$ such that

$$
\Delta t<\frac{1-e^{-\Delta t}}{u_{0}\left(1-e^{-t_{n}}\right)} .
$$

Since $t_{n} \leq T$, and $e^{T}<u_{0} /\left(u_{0}-1\right)$, we find that $u_{0}\left(1-e^{-t_{n}}\right) \leq u_{0}\left(1-e^{-T}\right)<1$. Thus we have to choose $\Delta t$ such that

$$
u_{0}\left(1-e^{-T}\right)<\frac{1}{\Delta t}\left(1-e^{-\Delta t}\right)=1-\frac{\Delta t}{2}+\mathcal{O}\left(\Delta t^{2}\right)
$$

or

$$
\Delta t<2\left(1-u_{0}\left(1-e^{-T}\right)\right) .
$$



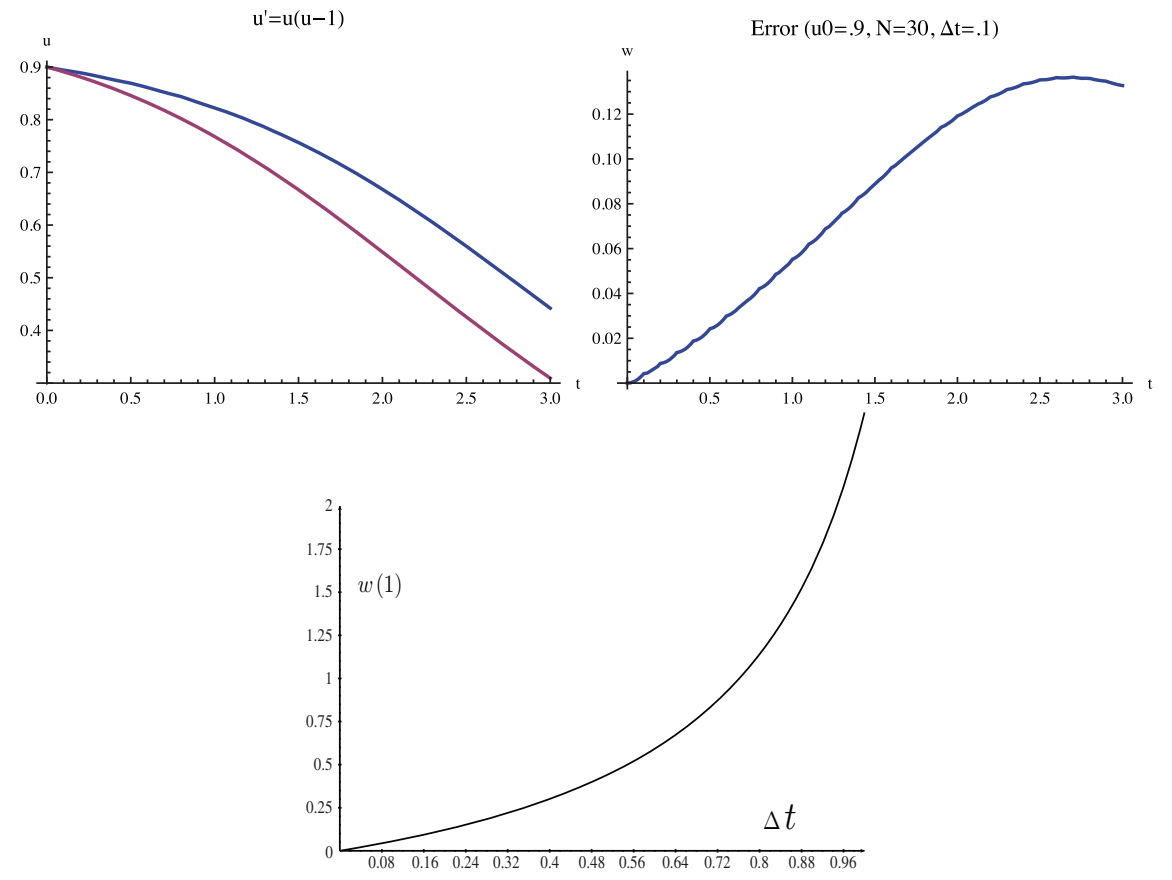

Figure 2. (Left) The exact (top) and the approximate (bottom) solution. (Right) The error. (Below) The error $w(1)$ as a function of $\Delta t$.

In this case one can verify (13) directly; namely,

$$
\begin{aligned}
& \left|v\left(t_{n}, t_{n}\right)-u\left(t_{n}\right)\right| \\
& \quad=\left|u_{0}\right|^{2}\left(e^{t_{n}}-1\right) \frac{\left|(\Delta t)^{-1}\left(1-e^{-\Delta t}\right)-1\right|}{\left|\left(u_{0}+e^{t_{n}}\left((\Delta t)^{-1}\left(1-e^{-\Delta t}\right)-u_{0}\right)\right)\left(u_{0}+e^{t_{n}}\left(1-u_{0}\right)\right)\right|} \\
& \quad=\mathcal{O}(\Delta t) .
\end{aligned}
$$

The example is illustrated in Figure 2

2.3. The KdV equation. Let us now apply this general framework to the KdV equation, that is,

$$
u_{t}=u u_{x}-u_{x x x},\left.\quad u\right|_{t=0}=u_{0} \in H^{s}(\mathbb{R}) .
$$

In this case, $C(u)=u u_{x}-u_{x x x}$, and the evolution operator $\Phi_{C}(t ; \cdot): H^{s} \rightarrow H^{s}$ is bounded. Therefore, the Hilbert space $\mathbb{R}^{n}$ of the previous example is replaced by the Sobolev space $H^{s}(\mathbb{R})$ with the inner product and norm

$$
(f, g)_{H^{s}(\mathbb{R})}=\sum_{j=0}^{s} \int_{\mathbb{R}} \partial_{x}^{j} f(x) \partial_{x}^{j} g(x) d x, \quad\|f\|_{H^{s}(\mathbb{R})}^{2}=(f, f)_{H^{s}(\mathbb{R})} .
$$

We choose $A$ to equal (minus) the Airy operator:

$$
\begin{aligned}
A(f) & =-f_{x x x}, \\
d A(f)[g] & =-g_{x x x}, \\
d^{(2)} A(f)[g, h] & =0,
\end{aligned}
$$


and $B$ to equal the Burgers operator:

$$
\begin{aligned}
B(f) & =f f_{x}, \\
d B(f)[g] & =f g_{x}+f_{x} g, \\
d^{(2)} B(f)[g, h] & =h g_{x}+h_{x} g, \\
d^{(3)} B(f)[g, h, k] & =0 .
\end{aligned}
$$

In this case the commutator reads

$$
[A, B](f, f)=-\frac{3}{2} \partial_{x}^{2}\left(f_{x}\right)^{2} .
$$

Thus the equations (17), (6), and (8) are

$$
\begin{aligned}
w_{t}-(u w)_{x}+w_{x x x} & =F+w w_{x}, \\
F & =v_{t}-v v_{x}, \\
F_{\tau}+F_{x x x} & =-\frac{3}{2} \partial_{x}^{2}\left(v_{x}\right)^{2},
\end{aligned}
$$

respectively.

From [2] we recall the classical result that for $u_{0} \in H^{s}(\mathbb{R})$ with $s \geq 2$ there exists a unique solution $u \in C\left([0, T], H^{s}(\mathbb{R})\right)$ of the $\mathrm{KdV}$ equation

$$
u_{t}=u u_{x}-u_{x x x},\left.\quad u\right|_{t=0}=u_{0} .
$$

In particular, we can assume that there exists a constant $K$ (depending on $T, u_{0}$, and $s$ ) such that

$$
\|u(t)\|_{H^{s}(\mathbb{R})} \leq K, \quad t \in[0, T] .
$$

To save space and typing efforts, we shall write $H^{k}$ for $H^{k}(\mathbb{R})$, and $\partial$ for $\partial_{x}$.

Next, let $s$ be an odd integer greater than or equal to 5 . Assume that there exists a constant $\alpha$ such that

$$
\|v(t, \tau)\|_{H^{\hat{k}}} \leq \alpha, \quad(t, \tau) \in \Omega_{\Delta t},
$$

where $\hat{k}(s)=(s-1) / 2$.

Let us estimate the behavior of the Airy and Burgers operators. The Airy equation leaves all Sobolev norms invariant, viz.

$$
\|v(t, \tau)\|_{H^{k}}=\left\|v\left(t, t_{n}\right)\right\|_{H^{k}} .
$$

By definition we find for the Burgers operator,

$$
\begin{aligned}
\frac{1}{2} \frac{d}{d t}\left\|v\left(t, t_{n}\right)\right\|_{H^{s}}^{2} & =\left(v, v_{t}\right)_{H^{s}}=\sum_{j=0}^{s} \int \partial^{j} v \partial^{j}\left(v v_{x}\right) d x \\
& =\sum_{j=0}^{s} \sum_{k=0}^{j}\left(\begin{array}{l}
j \\
k
\end{array}\right) \int \partial^{j} v \partial^{k+1} v \partial^{j-k} v d x .
\end{aligned}
$$

For $j<s$, any of the above terms can be estimated by

$$
\begin{aligned}
\left|\int \partial^{j} v \partial^{k+1} v \partial^{j-k} v d x\right| & \leq\left\|\partial^{j} v\right\|_{L^{\infty}}\left\|\partial^{\max \{k+1, j-k\}} v\right\|_{L^{2}}\left\|\partial^{\min \{k+1, j-k\}} v\right\|_{L^{2}} \\
& \leq K\|v\|_{H^{s}}^{2}\|v\|_{H^{\hat{k}}} \\
& \leq K_{\alpha}\|v\|_{H^{s}}^{2} .
\end{aligned}
$$


For $j=s$ all terms with $k<s$ and $k \neq \hat{k}$ can be estimated similarly,

$$
\begin{aligned}
\left|\int \partial^{s} v \partial^{k+1} v \partial^{s-k} v d x\right| & \leq\left\|\partial^{s} v\right\|_{L^{2}}\left\|\partial^{\min \{k+1, s-k\}} v\right\|_{L^{\infty}}\left\|\partial^{\max \{k+1, s-k\}} v\right\|_{L^{2}} \\
& \leq K\|v\|_{H^{s}}^{2}\|v\|_{H^{\hat{k}}} \\
& \leq K_{\alpha}\|v\|_{H^{s}}^{2} .
\end{aligned}
$$

If $j=s$ and $k=\hat{k}$, we find

$$
\begin{aligned}
\left|\int \partial^{s} v \partial^{\hat{k}+1} v \partial^{s-\hat{k}} v d x\right|= & \left|\int \partial^{s} v\left(\partial^{\hat{k}+1} v\right)^{2} d x\right|=2\left|\int \partial^{s-1} v \partial^{\hat{k}+1} v \partial^{\hat{k}+2} v d x\right| \\
= & 2\left|\int \partial^{\hat{k}} v \partial\left(\partial^{s-1} v \partial^{\hat{k}+2} v\right) d x\right| \\
\leq & 2 \int\left|\partial^{\hat{k}} v \partial^{s} v \partial^{\hat{k}+2} v\right| d x+2 \int\left|\partial^{\hat{k}} v \partial^{s-1} v \partial^{\hat{k}+3} v\right| d x \\
\leq & 2\left\|\partial^{\hat{k}+2} v\right\|_{L^{\infty}}\left\|\partial^{\hat{k}} v\right\|_{L^{2}}\left\|\partial^{s-1} v\right\|_{L^{2}} \\
& +2\left\|\partial^{\hat{k}+3} v\right\|_{L^{\infty}}\left\|\partial^{\hat{k}} v\right\|_{L^{2}}\left\|\partial^{s-1} v\right\|_{L^{2}} \\
\leq & 4 K\|v\|_{H^{s}}^{2}\|v\|_{H^{\hat{k}}} \\
\leq & K_{\alpha}\|v\|_{H^{s}}^{2} .
\end{aligned}
$$

We are left with the term where $k=s=j$, viz.

$$
\begin{aligned}
\left|\int \partial^{s} v \partial^{s+1} v v d x\right| & =\frac{1}{2}\left|\int\left(\partial^{s} v\right)^{2} \partial v d x\right| \\
& \leq\|\partial v\|_{L^{\infty}}\left\|\partial^{s} v\right\|_{L^{2}}^{2} \\
& \leq K\|v\|_{H^{\hat{k}}}\|v\|_{H^{s}}^{2}
\end{aligned}
$$

if $\hat{k} \geq 2$, i.e., if $s \geq 5$.

Thus

$$
\frac{d}{d t}\left\|v\left(t, t_{n}\right)\right\|_{H^{s}} \leq K_{\alpha}\left\|v\left(t, t_{n}\right)\right\|_{H^{s}},
$$

which implies that

$$
\left\|v\left(t, t_{n}\right)\right\|_{H^{s}} \leq e^{K_{\alpha}\left(t-t_{n}\right)}\left\|v\left(t_{n}, t_{n}\right)\right\|_{H^{s}} .
$$

In particular, for any $n$,

$$
\left\|v\left(t_{n}, t_{n}\right)\right\|_{H^{s}} \leq e^{K_{\alpha} \Delta t}\left\|v\left(t_{n-1}, t_{n-1}\right)\right\|_{H^{s}} \leq e^{K_{\alpha} t_{n}}\left\|u_{0}\right\|_{H^{s}} .
$$

Thus we have shown the following result.

Lemma 2.3. We have

$$
\|v(t, \tau)\|_{H^{s}} \leq K_{\alpha}, \quad(t, \tau) \in \Omega_{\Delta t} .
$$

Observe the general result, obtained by integration by parts,

$$
\left(f, f_{x x x}\right)_{H^{k}}=0, \quad f \in H^{k+3} .
$$

Next we analyze the forcing term that satisfies (15). By taking the $H^{s-3}$ inner product with $F$ in (15) we get

$$
\frac{1}{2} \partial_{\tau}\|F(t, \tau)\|_{H^{s-3}}^{2}=-\left(F, F_{x x x}\right)_{H^{s-3}}-\frac{3}{2}\left(F, \partial^{2}\left(v_{x}\right)^{2}\right)_{H^{s-3}}
$$




$$
\leq \frac{3}{2}\|F\|_{H^{s-3}}\left\|\partial_{x}^{j+2}\left(v_{x}\right)^{2}\right\|_{H^{s-3}} .
$$

Since $H^{s}$ is an algebra,

$$
\left\|\partial^{j+2}\left(v_{x}\right)^{2}\right\|_{H^{s-3}} \leq\left\|\left(v_{x}\right)^{2}\right\|_{H^{s-1}} \leq K\left\|v_{x}\right\|_{H^{s-1}}^{2} \leq K_{\alpha}^{2} .
$$

Also $F\left(t, t_{n}\right)=0$ for $t \in\left[t_{n}, t_{n+1}\right]$, and we conclude that

$$
\|F(t, \tau)\|_{H^{s-3}} \leq K_{\alpha} \Delta t, \quad(t, \tau) \in \Omega_{\Delta t} .
$$

As for the error function $w=v(t, t)-u(t)$, we have the following estimates. Let $E(t)=\|w(t)\|_{H^{s-3}}$. By taking the inner product with $w$ in (14), we get

$$
\begin{aligned}
\frac{1}{2} \frac{d}{d t} E^{2}(t) & =\left(w,(u w)_{x}\right)_{H^{s-3}}-\left(w, w_{x x x}\right)_{H^{s-3}}+(w, F)_{H^{s-3}}+\left(w, w w_{x}\right)_{H^{s-3}} \\
& =\left(w,(u w)_{x}\right)_{H^{s-3}}+\left(w, w w_{x}\right)_{H^{s-3}}+(w, F)_{H^{s-3}} \\
& =\sum_{j=0}^{s-3} \int\left(\partial^{j} w \partial^{j}\left(u w_{x}+u_{x} w+w w_{x}\right)\right) d x+(w, F)_{H^{s-3}} .
\end{aligned}
$$

The first integrand on the right is expanded by Leibniz' rule. We get

$$
\sum_{j=0}^{s-3} \sum_{k=0}^{j}\left(\begin{array}{l}
j \\
k
\end{array}\right) \int \partial^{j} w \partial^{k+1} w \partial^{j-k} u+\partial^{j} w \partial^{k} w \partial^{j+1-k} u+\partial^{j} w \partial^{k} w \partial^{j+1-k} w d x .
$$

For $0 \leq k \leq j<s-3$, we can estimate

$$
\begin{aligned}
\left|\int \partial^{j} w \partial^{k+1(k)} w \partial^{j-k(+1)} u d x\right| & \leq\|w\|_{H^{s-3}}^{2}\left\|\partial^{j-k(+1)} u\right\|_{L^{\infty}} \\
& \leq K\|w\|_{H^{s-3}}^{2}\|u\|_{H^{s}}, \\
\left|\int \partial^{j} w \partial^{k} w \partial^{j+1-k} w d x\right| & \leq\left\|\partial^{j} w\right\|_{L^{\infty}}\|w\|_{H^{s-3}}^{2} \\
& \leq K\left(\|u\|_{H^{s}}+\|v\|_{H^{s}}\right)\|w\|_{H^{s-3}}^{2} .
\end{aligned}
$$

For $j=s-3$, we can use the same strategy for those terms with fewer than $s-2$ derivatives on $w$. The term with $s-2$ derivatives on $w$ can be estimated as

$$
\begin{aligned}
\left|\int \partial^{s-3} w \partial^{s-2} w u d x\right| & =\frac{1}{2}\left|\int\left(\partial^{s-3} w\right)^{2} \partial u d x\right| \leq K\|u\|_{H^{s}}\|w\|_{H^{s-3}}^{2}, \\
\left|\int \partial^{s-3} w w \partial^{s-2} w d x\right| & =\frac{1}{2}\left|\int\left(\partial^{s-3} w\right)^{2} \partial w d x\right| \\
& \leq K\left(\|u\|_{H^{s}}+\|v\|_{H^{s}}\right)\|w\|_{H^{s-3}}^{2} .
\end{aligned}
$$

The last term in (17) is overestimated by $\|F\|_{H^{s-3}}\|w\|_{H^{s-3}}$, and we get

$$
\frac{d}{d t} E^{2}(t) \leq K_{\alpha} E^{2}(t)+K E(t)\|F\|_{H^{s-3}} \leq K_{\alpha} E^{2}(t)+K \Delta t
$$

which implies that

$$
\frac{d}{d t} E(t) \leq K_{\alpha} E(t)+K \Delta t .
$$

Since $E(0)=0$, Gronwall's inequality yields

$$
\|w(t)\|_{H^{s-3}}=E(t) \leq K_{\alpha} \Delta t .
$$


Recall that $\|v(t, \tau)\|_{H^{s-3}}=\|v(t, t)\|_{H^{s-3}}$ because

$$
\partial_{\tau}\|v(t, \tau)\|_{H^{s-3}}^{2}=-2\left(v, v_{x x x}\right)_{H^{s-3}}=0 .
$$

Now $u$ is bounded in $H^{s}$, and we infer that

$$
\|v(t, \tau)\|_{H^{s-3}} \leq K+K_{\alpha} \Delta t .
$$

Since $s \geq 5$,

$$
s-3 \geq \hat{k}=\frac{s-1}{2}
$$

and we get

$$
\|v(t, \tau)\|_{H^{\hat{k}}} \leq K+K_{\alpha} \Delta t .
$$

First choose $\alpha \geq 4 K$, then choose $\Delta t$ so small that $K_{\alpha} \Delta t \leq \alpha / 4$, so that $K+$ $K_{\alpha} \Delta t \leq \alpha / 4+\alpha / 4=\alpha / 2$. Hence, by the bootstrap argument we have proved the following theorem.

Theorem 2.4. Fix $T>0$. Let $u_{0} \in H^{s}(\mathbb{R})$ with $s \geq 5$. Then for $\Delta t$ sufficiently small we have

$$
\|v(t, t)-u(t)\|_{H^{s-3}(\mathbb{R})} \leq K \Delta t, \quad t \in[0, T],
$$

where $K$ depends on $s, T$ and $u_{0}$ only.

Remark 2.5. Instead of defining $v$ by (4) for the KdV equation, we could also interchange the order of the Airy operator $A$ and the Burgers operator $B$ in the definition of $v$. The same procedure as described above would apply, and Theorem 2.4 would remain valid. This remark is important for the Strang splitting to be discussed next.

\section{Strang SPLitTing}

To achieve higher-order convergence it is common to consider the so-called Strang splitting. Now we approximate the solution by using two Godunov splittings, each with a time step of $\Delta t / 2$, and in alternating order. Explicitly, we define

$$
\begin{aligned}
v(0,0) & =u_{0}, \\
v_{t}\left(t, t_{n}\right) & =B\left(v\left(t, t_{n}\right)\right), \quad t \in\left(t_{n}, t_{n+1 / 2}\right], \\
v_{\tau}(t, \tau) & =A(v(t, \tau)), \quad(t, \tau) \in\left[t_{n}, t_{n+1 / 2}\right] \times\left(t_{n}, t_{n+1 / 2}\right], \\
v_{\tau}\left(t_{n+1 / 2}, \tau\right) & =A\left(v\left(t_{n+1 / 2}, \tau\right)\right), \quad \tau \in\left(t_{n+1 / 2}, t_{n+1}\right], \\
v_{t}(t, \tau) & =B(v(t, \tau)), \quad(t, \tau) \in\left(t_{n+1 / 2}, t_{n+1}\right] \times\left[t_{n+1 / 2}, t_{n+1}\right],
\end{aligned}
$$

for $n=0, \ldots,\lfloor T / \Delta t\rfloor$. We consider this function for $(t, \tau)$ in the domain

$$
\widetilde{\Omega}_{\Delta t}=\bigcup_{n=0}^{\lfloor T / \Delta t\rfloor}\left(\left[t_{n}, t_{n+1 / 2}\right]^{2} \cup\left[t_{n+1 / 2}, t_{n+1}\right]^{2}\right) .
$$

The aim is now to show that

$$
\|v(t, t)-u(t)\|=\mathcal{O}\left(\Delta t^{2}\right)
$$

in an appropriate norm. Here the abstract analysis of Section 2.1 applies, and we find: 


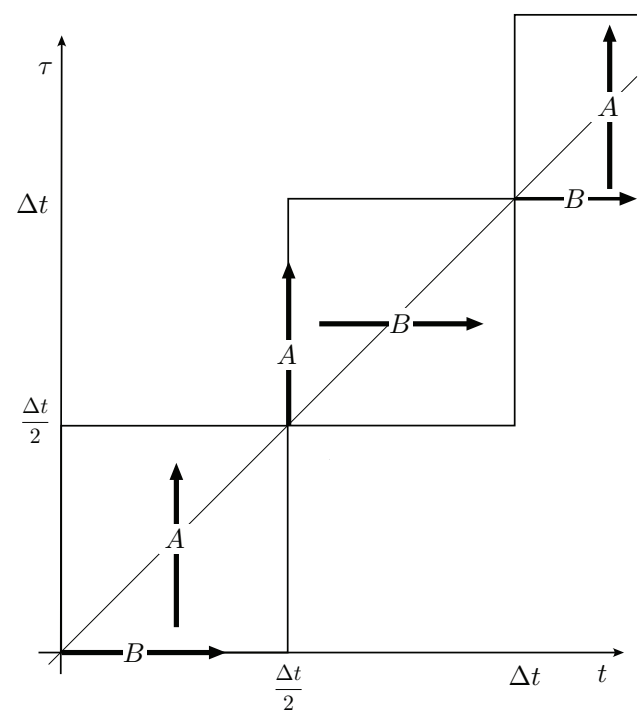

FiguRE 3. A schematic view of Strang splitting.

(i): On the domains $\left[t_{n}, t_{n+1 / 2}\right] \times\left[t_{n}, t_{n+1 / 2}\right]$ we have

$$
\begin{aligned}
w_{t}-d C(u)[w] & =F(t)+\int_{0}^{1}(1-\alpha) d^{(2)} C(u+\alpha w)[w]^{2} d \alpha, \\
F(t, \tau) & =v_{t}(t, \tau)-B(v(t, \tau)) .
\end{aligned}
$$

The forcing term satisfies the following time development:

$$
\begin{aligned}
F_{\tau} & =d A(v)[F]+[A, B](v), \\
F_{t} & =v_{t t}-d B(v)[F]-d B(v)[B(v)] .
\end{aligned}
$$

(ii): On the domains $\left[t_{n+1 / 2}, t_{n+1}\right] \times\left[t_{n+1 / 2}, t_{n+1}\right]$ we have (here we write $w_{t}$ rather than $w_{\tau}$ since $w$ is a function of one variable only)

$$
\begin{aligned}
w_{t}-d C(u)[w] & =G(t)+\int_{0}^{1}(1-\alpha) d^{(2)} C(u+\alpha w)[w]^{2} d \alpha, \\
G(t, \tau) & =v_{\tau}(t, \tau)-A(v(t, \tau)) .
\end{aligned}
$$

The forcing term satisfies the following time development:

$$
\begin{aligned}
G_{t} & =d B(v)[G]+[B, A](v), \\
G_{\tau} & =v_{\tau \tau}-d A(v)[G]-d A(v)[A(v)] .
\end{aligned}
$$

We extend $F$ and $G$ to all of $\widetilde{\Omega}_{\Delta t}$ using the same definitions, (19) and (20), respectively. Observe that this implies that $F=0$ on $\left[t_{n+1 / 2}, t_{n+1}\right] \times\left[t_{n+1 / 2}, t_{n+1}\right]$, while $G=0$ on $\left[t_{n}, t_{n+1 / 2}\right] \times\left[t_{n}, t_{n+1 / 2}\right]$. The total forcing term is defined by

$$
H(t, \tau)=F(t, \tau)+G(t, \tau) .
$$

3.1. Ordinary differential equations. One can consider the case of ordinary differential equations, as we did in Subsection 2.2 for Godunov splitting, but for reasons of brevity we will only revisit the example in Remark 2.2 . 

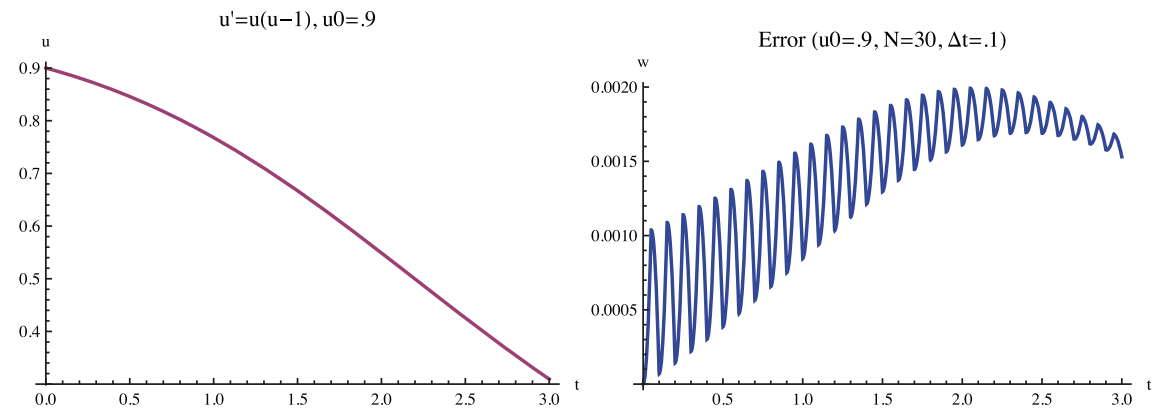

FiguRE 4. (Left) The exact and the approximate solution. (Right) The error $v(t, t)-u(t)$.

Remark 3.1. We find, using the definitions (18), that

$$
v(t, \tau)= \begin{cases}\frac{v\left(t_{n}, t_{n}\right) e^{-\left(\tau-t_{n}\right)}}{1-v\left(t_{n}, t_{n}\right)\left(t-t_{n}\right)}, & \text { for } t, \tau \in\left[t_{n}, t_{n+1 / 2}\right], \\ \frac{v\left(t_{n+1 / 2}, t_{n+1 / 2}\right)}{e^{\tau-t_{n+1 / 2}-v\left(t_{n+1 / 2}, t_{n+1 / 2}\right)\left(t-t_{n+1 / 2}\right)},} & \text { for } t, \tau \in\left[t_{n+1 / 2}, t_{n+1}\right],\end{cases}
$$

for $n=0, \ldots,\lfloor T / \Delta t\rfloor$. By induction we determine

$$
v\left(t_{n}, t_{n}\right)=\frac{u_{0}\left(1-e^{-\Delta t}\right)}{\left(1-e^{-\Delta t}\right) e^{t_{n}}+u_{0} \Delta t\left(e^{t_{n}}-1\right)\left(e^{\Delta t}+1\right) / 2} .
$$

In this case we compute, when we for convenience write $\alpha=u_{0}\left(1-e^{t_{n}}\right)$,

$$
\begin{aligned}
\left|w\left(t_{n}\right)\right| & =\left|v\left(t_{n}, t_{n}\right)-u\left(t_{n}\right)\right| \\
& =\left|\frac{u_{0}\left(1-e^{-\Delta t}\right)}{\left(1-e^{-\Delta t}\right) e^{t_{n}}+u_{0} \Delta t\left(e^{t_{n}}-1\right)\left(e^{\Delta t}+1\right) / 2}-\frac{u_{0}}{e^{t_{n}}+u_{0}\left(1-e^{t_{n}}\right)}\right| \\
& =\left|u_{0}\right|\left|\frac{1}{e^{t_{n}}+\alpha \Delta t\left(1+e^{\Delta t}\right) /\left(2\left(1-e^{-\Delta t}\right)\right)}-\frac{1}{e^{t_{n}}+\alpha}\right| \\
& \leq \frac{\left|u_{0}\right|^{2} e^{t_{n}}\left(e^{t_{n}}-1\right)}{\left|\left(e^{t_{n}}+\alpha \Delta t\left(1+e^{\Delta t}\right) /\left(2\left(1-e^{-\Delta t}\right)\right)\right)\left(e^{t_{n}}+\alpha\right)\right|}\left|1-\frac{\Delta t\left(1+e^{\Delta t}\right)}{2\left(1-e^{-\Delta t}\right)}\right| \\
& \leq \mathcal{O}\left(\Delta t^{2}\right) .
\end{aligned}
$$

The example is illustrated in Figure 4. Observe the strong oscillations in the error. It is these oscillations which prevent the error from growing too large.

3.2. The $\mathbf{K d V}$ equation. For the $\mathrm{KdV}$ equation, we use $B(v)=v v_{x}$ and $A(v)=$ $-v_{x x x}$, and the above analysis yields:

(i): On domains $\left[t_{n}, t_{n+1 / 2}\right] \times\left[t_{n}, t_{n+1 / 2}\right]$ we have

$$
\begin{aligned}
w_{t}-(u w)_{x}+w_{x x x} & =F(t)+w w_{x}, \\
F(t, \tau) & =v_{t}-v v_{x} .
\end{aligned}
$$

The forcing term satisfies the following time development:

$$
\begin{gathered}
F_{\tau}=-F_{x x x}-\frac{3}{2} \partial_{x}^{2}\left(v_{x}\right)^{2}, \\
F_{t}=v_{t t}-(v F)_{x}-\left(2 v v_{x}^{2}+v^{2} v_{x x}\right), \\
\text { since } d B(v)[B(v)]=v\left(v v_{x}\right)_{x}+v_{x}\left(v v_{x}\right)=2 v v_{x}^{2}+v^{2} v_{x x} .
\end{gathered}
$$


(ii): On domains $\left[t_{n+1 / 2}, t_{n+1}\right] \times\left[t_{n+1 / 2}, t_{n+1}\right]$ we have

$$
\begin{aligned}
w_{t}-(u w)_{x}+w_{x x x} & =G(t)+w w_{x}, \\
G(t, \tau) & =v_{\tau}(t, \tau)+v_{x x x} .
\end{aligned}
$$

The forcing term satisfies the following time development:

$$
\begin{aligned}
G_{t} & =(v G)_{x}+\frac{3}{2} \partial_{x}^{2}\left(v_{x}\right)^{2}, \\
G_{\tau} & =v_{\tau \tau}+G_{x x x}-v^{(6)},
\end{aligned}
$$

since $d A(v)[A(v)]=\left(v_{x x x}\right)_{x x x}$.

To start the bootstrapping procedure we fix an odd integer $s$ and a positive constant $\alpha$, whose values will be determined in the course of the argument. Now assume that

$$
\|v(t, \tau)\|_{H^{\hat{k}}} \leq \alpha, \quad(t, \tau) \in \widetilde{\Omega}_{\Delta t},
$$

where $\hat{k}=(s-1) / 2$. As a consequence, we have

$$
\|v(t, \tau)\|_{H^{s}} \leq K_{\alpha}, \quad(t, \tau) \in \widetilde{\Omega}_{\Delta t}
$$

cf. the proof of Lemma 2.3, which can be easily adapted to Strang splitting.

We need to introduce the function

$$
z(t)=w(t)+w\left(t+\frac{\Delta t}{2}\right)=w(t)+\tilde{w}(t) .
$$

In the following we will write $\tilde{\phi}(t)=\phi\left(t+\frac{\Delta t}{2}\right)$ for any function $\phi$. Straightforward calculations yield that $z$ satisfies

$$
z_{t}-\left(\frac{1}{2} z^{2}+u z-z_{x x}\right)_{x}=H+\widetilde{H}+(\tilde{w}(\tilde{u}-u)-w \tilde{w})_{x} .
$$

By our techniques, we must work in $H^{s-9}$ (so at least $s \geq 9$ ); therefore set

$$
E(t)=\|z(t)\|_{H^{s-9}(\mathbb{R})} .
$$

By taking the $H^{s-9}$ inner product with $z$ in (29) we get

$$
\begin{aligned}
\frac{1}{2} \frac{d}{d t} E^{2}(t)= & \left(z,\left(\frac{1}{2} z^{2}+u z-z_{x x}\right)_{x}\right)_{H^{s-9}}+(z, H+\widetilde{H})_{H^{s-9}} \\
& +\left(z,(\tilde{w}(\tilde{u}-u)-w \tilde{w})_{x}\right)_{H^{s-9}} \\
= & \left(z, z z_{x}+u_{x} z+u z_{x}\right)_{H^{s-9}}+(z, H+\widetilde{H})_{H^{s-9}} \\
& -\left(z_{x}, \tilde{w}(\tilde{u}-u)-w \tilde{w}\right)_{H^{s-9}} \\
\leq & \left(z, z z_{x}+u_{x} z+u z_{x}\right)_{H^{s-9}}+E(t)\|H+\widetilde{H}\|_{H^{s-9}} \\
& +E(t)\|\tilde{w}(\tilde{u}-u)-w \tilde{w}\|_{H^{s-9}} \\
\leq & K_{\alpha} E^{2}(t)+K E(t)\left(\|H+\widetilde{H}\|_{H^{s-9}}+\|\tilde{w}(\tilde{u}-u)-w \tilde{w}\|_{H^{s-9}}\right)
\end{aligned}
$$


Thus by Gronwall's inequality,

$$
\begin{gathered}
E(t) \leq E(0)+\int_{0}^{t} e^{K_{\alpha}(t-\sigma)}\left(\|H(\sigma, \sigma)+\widetilde{H}(\sigma, \sigma)\|_{H^{s-9}}\right. \\
\left.+\|\tilde{w}(\sigma)(\tilde{u}(\sigma)-u(\sigma))-w(\sigma) \tilde{w}(\sigma)\|_{H^{s-9}}\right) d \sigma \\
\leq E(0)+e^{K_{\alpha} t} \int_{0}^{t}\left(\|H(\sigma, \sigma)+\widetilde{H}(\sigma, \sigma)\|_{H^{s-9}}\right. \\
\left.\quad+\|\tilde{w}(\sigma)(\tilde{u}(\sigma)-u(\sigma))-w(\sigma) \tilde{w}(\sigma)\|_{H^{s-9}}\right) d \sigma .
\end{gathered}
$$

Next we turn to the detailed estimate of each of the terms in (30). We start with the most involved one, the forcing term, which can be estimated as follows. We consider the term $F$ first. Since $F\left(t, t_{n}\right)=0$, we easily see that $F_{t}\left(t_{n}, t_{n}\right)=0$; thus (cf. (25) $)\left(F_{t}+F_{\tau}\right)\left(t_{n}, t_{n}\right)=-\frac{3}{2} \partial_{x}^{2}\left(v_{x}\right)^{2}\left(t_{n}, t_{n}\right)$. Thus

$$
\begin{aligned}
F(t, t)= & \left(v_{t}-v v_{x}\right)(t, t) \\
= & F\left(t_{n}, t_{n}\right)+\left(F_{t}+F_{\tau}\right)\left(t_{n}, t_{n}\right)\left(t-t_{n}\right) \\
& +\frac{\left(t-t_{n}\right)^{2}}{2} \int_{0}^{1}\left(F_{t t}+2 F_{t \tau}+F_{\tau \tau}\right)\left(\sigma\left(t-t_{n}\right)+t_{n}, \sigma\left(t-t_{n}\right)+t_{n}\right) d \sigma \\
= & -\frac{3}{2} \partial_{x}^{2}\left(v_{x}\right)^{2}\left(t_{n}, t_{n}\right)\left(t-t_{n}\right) \\
& +\frac{\left(t-t_{n}\right)^{2}}{2} \int_{0}^{1}\left(F_{t t}+2 F_{t \tau}+F_{\tau \tau}\right)\left(\sigma\left(t-t_{n}\right)+t_{n}, \sigma\left(t-t_{n}\right)+t_{n}\right) d \sigma .
\end{aligned}
$$

As for the second derivatives, we find

$$
\begin{aligned}
F_{\tau \tau} & =-F_{\tau x x x}-\frac{3}{2} \partial_{x}^{2} \partial_{\tau}\left(v_{x}\right)^{2} \\
& =\partial_{x}^{6} F+\frac{3}{2} \partial_{x}^{5}\left(v_{x}\right)^{2}-3 \partial_{x}^{2}\left(v_{x} v^{(6)}\right), \\
F_{\tau t} & =-v_{t t x x x}-\left(v_{x x x} v_{x}+v v_{x x x x}\right)_{t}, \\
F_{t t} & =v_{t t t}-v_{t t} v_{x}-2 v_{t} v_{x t}-v v_{x t t} .
\end{aligned}
$$

Similarly we find for the forcing term $G$ the following estimates. Since $G\left(t_{n+1 / 2}, \tau\right)$ $=0$, we easily see that $G_{\tau}\left(t_{n+1 / 2}, t_{n+1 / 2}\right)=0$; thus (cf. (27)) we obtain $\left(G_{t}+G_{\tau}\right)\left(t_{n+1 / 2}, t_{n+1 / 2}\right)=\frac{3}{2} \partial_{x}^{2}\left(v_{x}\right)^{2}\left(t_{n+1 / 2}, t_{n+1 / 2}\right)$. Thus

$$
\begin{aligned}
& G\left(t+\frac{\Delta t}{2}, t+\frac{\Delta t}{2}\right)=\left(v_{\tau}+v_{x x x}\right)\left(t+\frac{\Delta t}{2}, t+\frac{\Delta t}{2}\right) \\
&= G\left(t_{n+1 / 2}, t_{n+1 / 2}\right)+\left(G_{t}+G_{\tau}\right)\left(t_{n+1 / 2}, t_{n+1 / 2}\right)\left(t-t_{n}\right) \\
&+\frac{\left(t-t_{n}\right)^{2}}{2} \int_{0}^{1}\left(G_{t t}+2 G_{t \tau}+G_{\tau \tau}\right)\left(\sigma\left(t-t_{n}\right)+t_{n}, \sigma\left(t-t_{n}\right)+t_{n}\right) d \sigma \\
&= \frac{3}{2} \partial_{x}^{2}\left(v_{x}\right)^{2}\left(t_{n+1 / 2}, t_{n+1 / 2}\right)\left(t-t_{n}\right) \\
&+\frac{\left(t-t_{n}\right)^{2}}{2} \int_{0}^{1}\left(G_{t t}+2 G_{t \tau}+G_{\tau \tau}\right)\left(\sigma\left(t-t_{n}\right)+t_{n}, \sigma\left(t-t_{n}\right)+t_{n}\right) d \sigma .
\end{aligned}
$$

The second derivatives $G_{t t}+2 G_{t \tau}+G_{\tau \tau}$ will have to be considered similarly to those for $F$. These read

$$
G_{t t}=2 v_{x}^{2} G+4 v v_{x} G_{x}+v^{2} G_{x x}+G v v_{x x}
$$




$$
\begin{gathered}
+6 v_{x x}\left(v_{x} v\right)_{x x}+v v_{x} v_{x x x}+v\left(v v_{x}\right)_{x x x}, \\
G_{\tau t}=v_{\tau \tau} v_{x}+2 v_{\tau} v_{\tau x}+v v_{\tau \tau x}+\partial^{4}(G v)+\frac{3}{2} \partial^{5}\left(v_{x}^{2}\right)-\partial^{6}\left(v v_{x}\right), \\
G_{\tau \tau}=v_{\tau \tau \tau}+v_{\tau \tau x x x} .
\end{gathered}
$$

Lemma 3.2. We have the estimate

$$
\|\Phi(t, \tau)\|_{H^{s-9}}^{2}+\|\Psi(\bar{t}, \bar{\tau})\|_{H^{s-9}}^{2} \leq K_{\alpha}, \quad(t, \tau),(\bar{t}, \bar{\tau}) \in \widetilde{\Omega}_{\Delta t},
$$

where $\Phi=\left(F_{t t}+2 F_{t \tau}+F_{\tau \tau}\right)$ and $\Psi=\left(G_{t t}+2 G_{t \tau}+G_{\tau \tau}\right)$.

Proof. We have that $\|F\|_{H^{s-9}} \leq K_{\alpha} \Delta t$; we shall get a similar estimate for $\|G\|_{H^{s-9}}$. For $t_{n} \leq \tau \leq t_{n+1 / 2}$, we have that

$$
G_{t}=v_{x} G+v G_{x}+\frac{3}{2} \partial^{2}\left(v_{x}^{2}\right),
$$

and $G\left(t, t_{n}\right)=0$. Taking the $H^{s-9}$ inner product with $G$ we get

$$
\frac{1}{2} \frac{d}{d t}\|G\|_{H^{s-9}}^{2}=\sum_{k=0}^{s-9} \int \partial^{k}\left(v_{x} G\right) \partial^{k} G+\partial^{k}\left(v G_{x}\right) \partial^{k} G+\frac{3}{2} \partial^{k+2}\left(v_{x}^{2}\right) \partial^{k} G d x .
$$

The first term expands by the Leibniz rule; a typical term in this expansion reads (here $0 \leq j \leq k \leq s-9$ )

$$
\int \partial^{j+1} v \partial^{k-j} G \partial^{k} G d x \leq\left\|\partial^{j+1} v\right\|_{L^{\infty}}\|G\|_{H^{s-9}}^{2} .
$$

Similarly the second term can be expanded and estimated, except for the term containing $\partial^{k+1} G$, which is estimated as

$$
\begin{aligned}
\int v \partial^{k+1} G \partial^{k} G d x=\frac{1}{2} \int v \partial\left(\partial^{k} G\right)^{2} d x & =-\frac{1}{2} \int \partial v\left(\partial^{k} G\right)^{2} d x \\
& \leq \frac{1}{2}\|\partial v\|_{L^{\infty}}\|G\|_{H^{s-9}}^{2} .
\end{aligned}
$$

We bound the last term as

$$
\left|\int \partial^{k+2}\left(v_{x}^{2}\right) \partial^{k} G d x\right| \leq K\|v\|_{H^{s}}^{2}\|G\|_{H^{s-9}} .
$$

Summing up, we get

$$
\frac{d}{d t}\|G\|_{H^{s-9}}^{2} \leq K_{\alpha}\|G\|_{H^{s-9}}^{2}+K_{\alpha}\|G\|_{H^{s-9}} .
$$

Using Gronwall's inequality and that $G\left(t_{n}, \tau\right)=0$ we get

$$
\|G\|_{H^{s-9}} \leq K_{\alpha} \Delta t
$$

We also need estimates for $v_{t}, v_{t t}$ and $v_{t t t}$, where $t, \tau \in\left[t_{n}, t_{n+1 / 2}\right]$. In this set, $v_{\tau}=-v_{x x x}$, and this evolution preserves the $H^{k}$ norm. For $\tau=t_{n}$,

$$
\begin{aligned}
v_{t} & =v v_{x}, \\
v_{t t} & =v_{t} v_{x}+v v_{t x}, \\
v_{t t t} & =v_{t t} v_{x}+2 v_{t} v_{t x}+v v_{t t x} .
\end{aligned}
$$


Therefore

$$
\begin{aligned}
\left\|v_{t}\right\|_{H^{k}} & \leq\|v\|_{H^{k}}\|v\|_{H^{k+1}} \leq K\|v\|_{H^{k+1}}^{2} \\
\left\|v_{t t}\right\|_{H^{k}} & \leq K\|v\|_{H^{k+2}}^{3} \\
\left\|v_{t t t}\right\|_{H^{k}} & \leq K\|v\|_{H^{k+3}}^{4}
\end{aligned}
$$

Next we turn to estimates of $v_{\tau}, v_{\tau \tau}$ and $v_{\tau \tau \tau}$ in the set $t, \tau \in\left[t_{n+1 / 2}, t_{n+1}\right]$. Here $v_{t}=v v_{x}$, and setting $\omega=v_{\tau}, \theta=v_{\tau \tau}$ and $\gamma=v_{\tau \tau \tau}$, we get

$$
\begin{aligned}
\omega_{t} & =v_{x} \omega+v \omega_{x}, & \omega\left(t_{n+1 / 2}, \tau\right) & =-\partial^{3} v, \\
\theta_{t} & =2 \omega \omega_{x}+v_{x} \theta+v \theta_{x}, & \theta\left(t_{n+1 / 2}, \tau\right) & =\partial^{6} v, \\
\gamma_{t} & =3 \theta \omega_{x}+3 \theta_{x} \omega+v_{x} \gamma+v \gamma_{x}, & \gamma\left(t_{n+1 / 2}, \tau\right) & =-\partial^{9} v .
\end{aligned}
$$

Starting with $\omega$, for $k<s-9$ we get

$$
\frac{1}{2} \frac{d}{d t}\|\omega\|_{H^{k}}^{2}=\sum_{j=0}^{k} \int \partial^{j}\left(v_{x} \omega\right) \partial^{j} \omega+\partial^{j}\left(v \omega_{x}\right) \partial^{j} \omega d x .
$$

Using Leibniz' rule, all terms except the ultimate one will be of the type

$$
\left|\int \partial^{\ell} v \partial^{j-\ell} \omega \partial^{j} d x\right| \leq\|v\|_{H^{s}}\|\omega\|_{H^{k}}^{2} .
$$

The ultimate term (with one too many derivatives on $\omega$ ) is as usual estimated as

$$
\left|\int v \partial^{k+1} \omega \partial^{k} \omega d x\right|=\frac{1}{2}\left|\int \partial v\left(\partial^{k} \omega\right)^{2} d x\right| \leq\|v\|_{H^{s}}\|\omega\|_{H^{k}}^{2} .
$$

Gronwall's inequality then yields

$$
\|\omega\|_{H^{k}}(t) \leq e^{K_{\alpha} \Delta t}\|\omega\|_{H^{k}}\left(t_{n+1 / 2}\right) \leq K_{\alpha}\|v\|_{H^{k+3}}\left(t_{n+1 / 2}, \tau\right) .
$$

Reasoning similarly for $\theta$, we find that

$$
\begin{aligned}
\|\theta\|_{H^{k}}(t) & \leq e^{K_{\alpha} \Delta t}\left(\max _{s \in\left[t_{n+1 / 2}, t_{n+1}\right]}\|\omega\|_{H^{k+1}}^{2}(s)+\|\theta\|_{H^{k}}\left(t_{n+1 / 2}\right)\right) \\
& \leq K_{\alpha}\left(\|v\|_{H^{k+4}}^{2}+\|v\|_{H^{k+6}}\right)\left(t_{n+1 / 2}, \tau\right) .
\end{aligned}
$$

Finally, the estimate for $\gamma$ reads

$$
\begin{aligned}
\|\gamma\|_{H^{k}} & \leq K_{\alpha}\left(\|\theta\|_{H^{k+1}}\|\omega\|_{H^{k+1}}+\|\gamma\|_{H^{k}}\right)\left(t_{n+1 / 2}, \tau\right) \\
& \leq K_{\alpha}\left(\|v\|_{H^{k+4}}^{2}+\|v\|_{H^{k+5}}^{4}+\|v\|_{H^{k+7}}^{2}+\|v\|_{H^{k+9}}\right)\left(t_{n+1 / 2}, \tau\right) .
\end{aligned}
$$

Summing up, we get

$$
\left\|v_{\tau}\right\|_{H^{k}} \leq K_{\alpha}\|v\|_{H^{k+3}},\left\|v_{\tau \tau}\right\|_{H_{k}} \leq K_{\alpha}\|v\|_{H^{k+6}},\left\|v_{\tau \tau \tau}\right\|_{H_{k}} \leq K_{\alpha}\|v\|_{H^{k+9}} .
$$

Now

$$
\|\Phi\|_{H^{s-9}} \leq\left\|F_{\tau \tau}\right\|_{H^{s-9}}+2\left\|F_{t \tau}\right\|_{H^{s-9}}+\left\|F_{t t}\right\|_{H^{s-9}} .
$$

Working in the square $\left[t_{n}, t_{n+1 / 2}\right]^{2}$, from (31),

$$
\begin{aligned}
\left\|F_{\tau \tau}\right\|_{H^{s-9}} & \leq\left\|\partial^{6} F\right\|_{H^{s-9}}+\frac{3}{2}\left\|\partial^{5}(\partial v)^{2}\right\|_{H^{s-9}}+3\left\|\partial^{2}\left(\partial v \partial^{6} v\right)\right\|_{H^{s-9}} \\
& \leq\|F\|_{H^{s-3}}+K_{\alpha}\|v\|_{H^{s-3}}^{2}+K_{\alpha}\|v\|_{H^{s-1}}^{2} \\
& \leq K_{\alpha}
\end{aligned}
$$


and

$$
\begin{gathered}
\left\|F_{t \tau}\right\|_{H^{s-9}} \leq\left\|v_{t t}\right\|_{H^{s-6}}+K_{\alpha}\left(\|v\|_{H^{s-8}}\left\|v_{t}\right\|_{H^{s-6}}+\left\|v_{t}\right\|_{H^{s-9}}\|v\|_{H^{s-6}}\right. \\
\left.+\|v\|_{H^{s-9}}\left\|v_{t}\right\|_{H^{s-5}}+\left\|v_{t}\right\|_{H^{s-9}}\|v\|_{H^{s-5}}\right) \\
\leq K_{\alpha}\left(\|v\|_{H^{s-4}}+\|v\|_{H^{s-8}}\|v\|_{H^{s-5}}\right. \\
+\|v\|_{H^{s-7}}\|v\|_{H^{s-7}}+\|v\|_{H^{s-9}}\|v\|_{H^{s-4}} \\
\left.\quad+\|v\|_{H^{s-8}}\|v\|_{H^{s-5}}\right) \\
\leq K_{\alpha},
\end{gathered}
$$

and also

$$
\begin{aligned}
\left\|F_{t t}\right\|_{H^{s-9}} & \leq\left\|v_{t t t}\right\|_{H^{s-9}}+\left\|v_{t t} v_{x}\right\|_{H^{s-9}}+2\left\|v_{t} v_{x x}\right\|_{H^{s-9}}+\left\|v v_{t x x}\right\|_{H^{s-9}} \\
& \leq K_{\alpha}\left(\|v\|_{H^{s-6}}+\|v\|_{H^{s-7}}\|v\|_{H^{s-8}}+\|v\|_{H^{s-9}}\|v\|_{H^{s-6}}\right) \\
& \leq K_{\alpha} .
\end{aligned}
$$

Hence $\|\Phi\|_{H^{s-9}} \leq K_{\alpha}$.

In the second square $\left[t_{n+1 / 2}, t_{n}\right]^{2}$, we write

$$
\|\Psi\|_{H^{s-9}} \leq\left\|G_{t t}\right\|_{H^{s-9}}+2\left\|G_{t \tau}\right\|_{H^{s-9}}+\left\|G_{\tau \tau}\right\|_{H^{s-9}} .
$$

Each term above is estimated individually as

$$
\begin{aligned}
\left\|G_{t t}\right\|_{H^{s-9}} \leq 2\left\|v_{x}^{2} G\right\|_{H^{s-9}}+4\left\|v v_{x} G_{x}\right\|_{H^{s-9}}+\left\|v^{2} G_{x x}\right\|_{H^{s-9}} & \\
& +3\left\|v_{x x}\left(v^{2}\right)_{x x}\right\|_{H^{s-9}}+\left\|v v_{x x} v_{x x x}\right\|_{H^{s-9}}+\left\|v\left(v v_{x}\right)_{x x x}\right\|_{H^{s-9}} \\
\leq & K_{\alpha}\left(\|v\|_{H^{s-8}}\|G\|_{H^{s-9}}+\|v\|_{H^{s-8}}\|G\|_{H^{s-8}}+\|v\|_{H^{s-9}}\|G\|_{H^{s-7}}\right. \\
& \left.\quad+\|v\|_{H^{s-7}}^{2}+\|v\|_{H^{s-6}}^{3}+\|v\|_{H^{s-5}}^{3}\right) \\
\leq & K_{\alpha},
\end{aligned}
$$

$$
\begin{aligned}
\left\|G_{t \tau}\right\|_{H^{s-9}} \leq & \left\|v_{\tau \tau} v_{x}\right\|_{H^{s-9}}+2\left\|v_{\tau} v_{x x}\right\|_{H^{s-9}}+\left\|v v_{\tau \tau x}\right\|_{H^{s-9}}+\left\|\partial^{4}(G v)\right\|_{H^{s-9}} \\
& \quad+\frac{3}{2}\left\|\partial^{5}\left(v_{x}^{2}\right)\right\|_{H^{s-9}}+\left\|\partial^{6}\left(v v_{x}\right)\right\|_{H^{s-9}} \\
\leq & K_{\alpha}\left(\|v\|_{H^{s-3}}\|v\|_{H^{s-8}}+\|v\|_{H^{s-6}}\|v\|_{H^{s-8}}+\|v\|_{H^{s-9}}\|v\|_{H^{s-1}}\right. \\
& \left.\quad+\|G\|_{H^{s-5}}\|v\|_{H^{s-5}}+\|v\|_{H^{s-3}}^{2}+\|v\|_{H^{s-3}}\|v\|_{H^{s-2}}\right) \\
\leq & \quad K_{\alpha},
\end{aligned}
$$

$$
\begin{aligned}
\left\|G_{\tau \tau}\right\|_{H^{s-9}} & \leq\left\|v_{\tau \tau \tau}\right\|_{H^{s-9}}+\left\|v_{\tau \tau x x x}\right\|_{H^{s-9}} \\
& \leq K_{\alpha}\left(\|v\|_{H^{s}}+\|v\|_{H^{s}}\right) \\
& \leq K_{\alpha} .
\end{aligned}
$$

Collecting (34), (33), (32), (35), (36) and (37) finishes the proof of the lemma.

Lemma 3.3. The map

$$
[0, T] \ni t \mapsto \partial^{2}\left(v_{x}^{2}\right)(t, t) \in H^{s-9}
$$

is Lipschitz continuous with Lipschitz constant at most $K_{\alpha}$. 
Proof. Set $w(t, \tau)=\partial^{2}\left(v_{x}^{2}\right)(t, \tau)$. Then we have

$$
\begin{gathered}
w_{t}=4 v_{x x} v_{t x x}+2 v_{t x x x} v_{x}+2 v_{x x x} v_{t x}, \\
w_{\tau}=4 v_{x x} v_{\tau x x}+2 v_{\tau x x x} v_{x}+2 v_{x x x} v_{\tau x} .
\end{gathered}
$$

In the square $\left[t_{n}, t_{n+1 / 2}\right]^{2}$ we have $v_{\tau}=-v_{x x x}$; thus

$$
w_{\tau}=-4 v_{x x} \partial^{5} v-2 \partial^{6} v v_{x}-2 v_{x x x} \partial^{4} v .
$$

Hence in this square,

$$
\begin{aligned}
\left\|w_{t}+w_{\tau}\right\|_{H^{s-9}} \leq K( & \|v\|_{H^{s-7}}\left\|v_{t}\right\|_{H^{s-7}}+\left\|v_{t}\right\|_{H^{s-6}}\|v\|_{H^{s-8}} \\
& +\|v\|_{H^{s-6}}\left\|v_{t}\right\|_{H^{s-8}}+\|v\|_{H^{s-7}}\|v\|_{H^{s-4}} \\
& \left.+\|v\|_{H^{s-3}}\|v\|_{H^{s-1}}+\|v\|_{H^{s-6}}\|v\|_{H^{s-5}}\right) \\
\leq K_{\alpha} . &
\end{aligned}
$$

In the second square $\left[t_{n+1 / 2}, t_{n+1}\right]^{2}$ we have $w_{t}=v v_{x}$, and

$$
w_{t}=4 v_{x x}\left(v v_{x}\right)_{x x}+2\left(v v_{x}\right)_{x x x} v_{x}+2 v_{x x x}\left(v v_{x}\right)_{x} .
$$

Therefore in this square,

$$
\begin{gathered}
\left\|w_{t}+w_{\tau}\right\|_{H^{s-9}} \leq K\left(\|v\|_{H^{s-7}}\left\|v v_{x}\right\|_{H^{s-7}}+\left\|v v_{x}\right\|_{H^{s-6}}\|v\|_{H^{s-9}}\right. \\
+\|v\|_{H^{s-6}}\left\|v v_{x}\right\|_{H^{s-8}}+\|v\|_{H^{s-8}}\left\|v_{\tau}\right\|_{H^{s-8}} \\
\left.+\|v\|_{H^{s-8}}\left\|v_{\tau}\right\|_{H^{s-6}}+\|v\|_{H^{s-6}}\left\|v_{\tau}\right\|_{H^{s-8}}\right) \\
\leq K\left(\|v\|_{H^{s-7}}^{2}\|v\|_{H^{s-6}}+\|v\|_{H^{s-7}}\|v\|_{H^{s-6}}\|v\|_{H^{s-9}}\right. \\
+\|v\|_{H^{s-6}}\|v\|_{H^{s-8}}\|v\|_{H^{s-7}}+\|v\|_{H^{s-8}}\|v\|_{H^{s-5}} \\
\left.+\|v\|_{H^{s-8}}\|v\|_{H^{s-3}}+\|v\|_{H^{s-6}}\|v\|_{H^{s-5}}\right) \\
\leq K_{\alpha} .
\end{gathered}
$$

From this lemma it follows that

$$
\Gamma(t)=-\partial^{2}\left(v_{x}\right)^{2}(t, t)+\partial^{2}\left(v_{x}\right)^{2}\left(t+\frac{\Delta t}{2}, t+\frac{\Delta t}{2}\right)
$$

satisfies

$$
\|\Gamma(t)\|_{H^{s-9}} \leq K_{\alpha} \Delta t, \quad t \in[0, T] .
$$

The following lemma will be convenient.

Lemma 3.4. For $t \in\left[t_{m}, t_{m+1 / 2}\right]$ we have

$$
\begin{aligned}
& \sum_{j=0}^{s-9} \int\left(\partial^{j}(F(t)+G(t+\Delta t / 2))\right)^{2} d x \leq K_{\alpha} \Delta t^{4}, \\
& \sum_{j=0}^{s-9} \int\left(\partial^{j}(F(t)+G(t-\Delta t / 2))\right)^{2} d x \leq K_{\alpha} \Delta t^{4} .
\end{aligned}
$$


Proof. We show that (38), (39) is proved similarly. By a Taylor expansion, for $t \in\left[t_{m}, t_{m+1 / 2}\right]$

$$
\begin{aligned}
\sum_{j=0}^{s-9} \int & \left(\partial^{j}(F(t)+G(t+\Delta t / 2))\right)^{2} d x \\
= & \sum_{j=0}^{s-9} \int\left[\partial ^ { j } \left(-\frac{3}{2} \partial^{2}\left(v_{x}\right)^{2}\left(t_{m}\right)\left(t-t_{m}\right)+\frac{\left(t-t_{m}\right)^{2}}{2} \int_{0}^{1} \Phi\left(\sigma\left(t-t_{m}\right)+t_{m}\right) d \sigma\right.\right. \\
& \left.\left.+\frac{3}{2} \partial^{2}\left(v_{x}\right)^{2}\left(t_{m}+\Delta t / 2\right)\left(t-t_{m}\right)+\frac{\left(t-t_{m}\right)^{2}}{2} \int_{0}^{1} \Psi\left(\sigma\left(t-t_{m}\right)+t_{m}\right) d \sigma\right)\right]^{2} d x \\
= & \sum_{j=0}^{s-9} \int\left[\partial ^ { j } \left(-\frac{3}{2} \partial^{2}\left(v_{x}\right)^{2}\left(t_{m}\right)+\frac{3}{2} \partial^{2}\left(v_{x}\right)^{2}\left(t_{m}+\Delta t / 2\right)\left(t-t_{m}\right)\right.\right. \\
& \left.\left.+\frac{\left(t-t_{m}\right)^{2}}{2} \int_{0}^{1}\left(\Phi\left(\sigma\left(t-t_{m}\right)+t_{m}\right)+\Psi\left(\sigma\left(t-t_{m}\right)+t_{m}\right)\right) d \sigma\right)\right]^{2} d x \\
\leq & 2 \frac{9}{4} \sum_{j=0}^{s-9} \int\left(\partial^{j}\left(-\partial^{2}\left(v_{x}\right)^{2}\left(t_{m}\right)+\partial^{2}\left(v_{x}\right)^{2}\left(t_{m}+\Delta t / 2\right)\right)\left(t-t_{m}\right)\right)^{2} d x \\
& +2 \sum_{j=0}^{s-9} \int\left(\partial^{j} \int_{0}^{1}\left(\Phi\left(\sigma\left(t-t_{m}\right)+t_{m}\right)+\Psi\left(\sigma\left(t-t_{m}\right)+t_{m}\right)\right) d \sigma\left(t-t_{m}\right)^{2}\right)^{2} d x \\
= & \frac{9}{2}\|\Gamma(t)\|_{H^{s-9}}^{2}\left(t-t_{m}\right)^{2} \\
& +2 \sum_{j=0}^{s-9} \int\left(\partial^{j} \int_{0}^{1}\left(\Phi\left(\sigma\left(t-t_{m}\right)+t_{m}\right)+\Psi\left(\sigma\left(t-t_{m}\right)+t_{m}\right)\right) d \sigma\right)^{2} d x\left(t-t_{m}\right)^{4} \\
\leq & K_{\alpha} \Delta t^{2}\left(t-t_{m}\right)^{2} \\
& +4 \int_{0}^{1}\left(\left\|\Phi\left(\sigma\left(t-t_{m}\right)+t_{m}\right)\right\|_{H^{s-9}}^{2}+\left\|\Psi\left(\sigma\left(t-t_{m}\right)+t_{m}\right)\right\|_{H^{s-9}}^{2}\right) d \sigma\left(t-t_{m}\right)^{4} \\
\leq & K_{\alpha} \Delta t^{2}\left(t-t_{m}\right)^{2}+K_{\alpha}\left(t-t_{m}\right)^{4} \\
\leq & K_{\alpha} \Delta t^{4} .
\end{aligned}
$$

Combining the above results, we find that for $t \in\left[t_{n-1}, t_{n}\right)$,

$$
\begin{gathered}
\int_{0}^{t}\|H(\sigma)+\widetilde{H}(\sigma)\|_{H^{s-9}} d \sigma \leq \int_{0}^{t_{n}}\|H(\sigma)+\widetilde{H}(\sigma)\|_{H^{s-9}} d \sigma \\
=\sum_{m=0}^{n-1} \int_{t_{m}}^{t_{m+1}}\left(\sum _ { j = 0 } ^ { s - 9 } \int \left(\partial^{j}(F(\sigma)+G(\sigma))\right.\right. \\
\left.\left.+\partial^{j}\left(F\left(\sigma+\frac{\Delta t}{2}\right)+G\left(\sigma+\frac{\Delta t}{2}\right)\right)\right)^{2} d x\right)^{1 / 2} d \sigma \\
=\sum_{m=0}^{n-1} \int_{t_{m}}^{t_{m+1}}\left(\sum _ { j = 0 } ^ { s - 9 } \int \left(\partial^{j}\left(F(\sigma)+G\left(\sigma+\frac{\Delta t}{2}\right)\right)\right.\right. \\
\left.\left.+\partial^{j}\left(F\left(\sigma+\frac{\Delta t}{2}\right)+G(\sigma)\right)\right)^{2} d x\right)^{1 / 2} d \sigma
\end{gathered}
$$




$$
\begin{aligned}
& \leq \sqrt{2} \sum_{m=0}^{n-1} \int_{t_{m}}^{t_{m+1}}\left(\sum _ { j = 0 } ^ { s - 9 } \int \left(\left(\partial^{j}\left(F(\sigma)+G\left(\sigma+\frac{\Delta t}{2}\right)\right)\right)^{2}\right.\right. \\
& \left.+\left(\partial^{j}\left(F\left(\sigma+\frac{\Delta t}{2}\right)+G(\sigma)\right)\right)^{2} d x\right)^{1 / 2} d \sigma \\
& \leq \sqrt{2} \sum_{m=0}^{n-1} \int_{t_{m}}^{t_{m+1}}\left[\left(\sum_{j=0}^{s-9} \int\left(\partial^{j}\left(F(\sigma)+G\left(\sigma+\frac{\Delta t}{2}\right)\right)\right)^{2} d x\right)^{1 / 2}\right. \\
& \left.+\left(\sum_{j=0}^{s-9} \int\left(\partial^{j}\left(F\left(\sigma+\frac{\Delta t}{2}\right)+G(\sigma)\right)\right)^{2} d x\right)^{1 / 2}\right] d \sigma \\
& =\sqrt{2} \sum_{m} \int_{t_{m}}^{t_{m+1 / 2}}\left(\sum_{j=0}^{s-9} \int\left(\partial^{j}(F(\sigma)+G(\sigma+\Delta t / 2))\right)^{2} d x\right)^{1 / 2} d \sigma \\
& +\int_{t_{m+1}}^{t_{m+3 / 2}}\left(\sum_{j=0}^{s-9} \int\left(\partial^{j}(F(\sigma)+G(\sigma-\Delta t / 2))\right)^{2} d x\right)^{1 / 2} d \sigma \\
& \leq K_{\alpha} \Delta t^{2}\left(\sum_{m} \int_{t_{m}}^{t_{m+1 / 2}} d \sigma+\sum_{m} \int_{t_{m}+1}^{t_{m+3 / 2}} d \sigma\right) \\
& \leq K_{\alpha} \Delta t^{2} \text {, }
\end{aligned}
$$

where we have used Lemma 3.4. This finishes the estimate for the forcing term.

Next we estimate the term $\int_{0}^{t}\|\tilde{w}(\sigma)(\tilde{u}(\sigma)-u(\sigma)) w(\sigma) \tilde{w}(\sigma)\|_{H^{s-9}} d \sigma$ in (30). Here we can use the estimates from the Godunov splitting to infer that

$$
\|w(\sigma)\|_{H^{s-9}}+\|\tilde{w}(\sigma)\|_{H^{s-9}} \leq K_{\alpha} \Delta t, \quad \sigma \in[0, T] .
$$

From the KdV equation we infer immediately

$$
\|\tilde{u}(\sigma)-u(\sigma)\|_{H^{s-9}} \leq \int_{0}^{\Delta t / 2}\left\|\left(u u_{x}-u_{x x x}\right)(\sigma+\tau)\right\|_{H^{s-9}} d \tau \leq K \Delta t, \quad \sigma \in[0, T] .
$$

Thus

$$
\begin{aligned}
& \int_{0}^{t}\|\tilde{w}(\sigma)(\tilde{u}(\sigma)-u(\sigma))-w(\sigma) \tilde{w}(\sigma)\|_{H^{s-9}} d \sigma \\
& \quad \leq K \int_{0}^{t}\left(\|\tilde{w}(\sigma)\|_{H^{s-9}}\|(\tilde{u}-u)(\sigma)\|_{H^{s-9}}+\|w(\sigma)\|_{H^{s-9}}\|\tilde{w}(\sigma)\|_{H^{s-9}}\right) d \sigma \\
& \quad \leq K_{\alpha} \Delta t^{2}
\end{aligned}
$$

The last term to estimate in (30) is $E(0)=w(0)+\tilde{w}(0)=w(\Delta t / 2)$. For $t \leq \Delta t / 2$, we find that

$$
\begin{aligned}
w(t)= & v(t, t)-u(t)=t \int_{0}^{1}(B(v(s t, 0))+A(v(t, s t))-C(u(s t))) d s \\
=t & \int_{0}^{1}\left(B(u(s t))+\int_{0}^{1} d B(u(s t)+\sigma(v(s t, 0)-u(s t)))[v(s t, 0)-u(s t)] d \sigma\right. \\
& +A(u(s t))+\int_{0}^{1} d A(u(s t)+\sigma(v(t, s t)-u(s t)))[v(t, s t)-u(s t)] d \sigma \\
& -(A+B)(u(s t))) d s
\end{aligned}
$$




$$
\begin{aligned}
& =t \int_{0}^{1} \int_{0}^{1}(d B(u(s t)+\sigma(v(s t, 0)-u(s t)))[v(s t, 0)-u(s t)] \\
& \quad+d A(u(s t)+\sigma(v(t, s t)-u(s t)))[v(t, s t)-u(s t)]) d s d \sigma \\
& =t \int_{0}^{1} \int_{0}^{1}\left(d B(u(s t)+\sigma(v(s t, 0)-u(s t)))\left[\int_{0}^{s t} \frac{d}{d \tau}(v(\tau, 0)-u(\tau)) d \tau\right]\right. \\
& \quad+d A(u(s t)+\sigma(v(t, s t)-u(s t)))\left[\int_{0}^{s t} \frac{d}{d \tau}(v(t, \tau)-u(\tau)) d \tau\right. \\
& \left.\left.\quad+\int_{0}^{t} \frac{d}{d \tau} v(\tau, 0) d \tau\right]\right) d s d \sigma \\
& =t \int_{0}^{1} \int_{0}^{1}\left[\int_{0}^{s t}(d B(u(s t)+\sigma(v(s t, 0)-u(s t)))[B(v(\tau, 0))-(A+B)(u(\tau))]\right. \\
& \quad+d A(u(s t)+\sigma(v(t, s t)-u(s t)))[B(v(t, \tau))-(A+B)(u(\tau))]) d \tau \\
& \left.\quad+\int_{0}^{t} d A(u(s t)+\sigma(v(t, s t)-u(s t)))[B(v(\tau, 0))] d \tau\right] d s d \sigma \\
& =t \int_{0}^{1} \int_{0}^{1}\left[\int _ { 0 } ^ { s t } \left(( u ( s t ) + \sigma ( v ( s t , 0 ) - u ( s t ) ) ) \left(v(\tau, 0) v(\tau, 0)_{x}\right.\right.\right. \\
& \left.\quad+\left(v(t, \tau) v(t, \tau)_{x}-u(\tau) u(\tau)_{x}+u(\tau)_{x x x}\right)_{x x x}\right) d \tau \\
& \left.+\int_{0}^{t}\left(v(\tau, 0) v(\tau, 0)_{x}\right)_{x x x} d \tau\right] d s d \sigma .
\end{aligned}
$$

Taking the $H^{s-9}$ norm above, using the triangle inequality, the bounds on $v$ and $u$ and the Cauchy-Schwarz inequality, we find that each of the above integrands is bounded by $K_{\alpha}$. Thus

$$
\begin{aligned}
\|w(t)\|_{H^{s-9}} & \leq t \int_{0}^{1} \int_{0}^{1}\left[\int_{0}^{s t} K_{\alpha} d \tau+\int_{0}^{t} K_{\alpha} d \tau\right] d s d \sigma \\
& \leq K_{\alpha} t^{2}
\end{aligned}
$$

Hence we infer that

$$
E(0)=\|w(\Delta t / 2)\|_{H^{s-9}} \leq K_{\alpha} \Delta t^{2} .
$$

Collecting the estimates from (40), (41), and (42), we find that (30) reads

$$
E(t)=\|z(t)\|_{H^{s-9}} \leq K_{\alpha} \Delta t^{2} .
$$

By the triangle inequality,

$$
\|w(t)\|_{H^{s-9}} \leq 2\|w(t)\|_{H^{s-9}} \leq\|z(t)\|_{H^{s-9}}+\|w(t)-\tilde{w}(t)\|_{H^{s-9}} .
$$

To estimate the last term on the right-hand side we write:

$$
\begin{array}{r}
\|\tilde{w}(t)-w(t)\|_{H^{s-9}} \leq\left\|w\left(t_{n}\right)-w\left(t_{n+1 / 2}\right)\right\|_{H^{s-9}}+\left\|w(t)-w\left(t_{n}\right)\right\|_{H^{s-9}} \\
+\left\|w(t+\Delta t / 2)-w\left(t_{n+1 / 2}\right)\right\|_{H^{s-9}},
\end{array}
$$


for $t \in\left[t_{n}, t_{n+1 / 2}\right]$. (Similar expressions hold when $t \in\left[t_{n+1 / 2}, t_{n+1}\right]$.) We note that

$$
\begin{aligned}
w\left(t_{n+1 / 2}\right) & =\Phi_{A}\left(\frac{\Delta t}{2}\right) \Phi_{B}\left(\frac{\Delta t}{2}\right) v\left(t_{n}, t_{n}\right)-\Phi_{C}\left(\frac{\Delta t}{2}\right) u\left(t_{n}\right), \\
w(t) & =\Phi_{A}\left(t-t_{n}\right) \Phi_{B}\left(t-t_{n}\right) v\left(t_{n}, t_{n}\right)-\Phi_{C}\left(t-t_{n}\right) u\left(t_{n}\right), \\
w\left(t+\frac{\Delta t}{2}\right) & =\Phi_{A}\left(t-t_{n}\right) \Phi_{B}\left(t-t_{n}\right) v\left(t_{n+1 / 2}, t_{n+1 / 2}\right)-\Phi_{C}\left(\frac{\Delta t}{2}\right) u\left(t_{n+1 / 2}\right),
\end{aligned}
$$

when $t \in\left[t_{n}, t_{n+1 / 2}\right]$. Each of the expressions on the right-hand side of (45) needs to be estimated:

$$
\begin{aligned}
w\left(t_{n+1 / 2}\right)-w\left(t_{n}\right)=\Phi_{A}\left(\frac{\Delta t}{2} ; \Phi_{B}\left(\frac{\Delta t}{2} ; v\left(t_{n}, t_{n}\right)\right)\right) \\
\quad-\Phi_{C}\left(\frac{\Delta t}{2} ; u\left(t_{n}\right)\right)-\left(v\left(t_{n}, t_{n}\right)-u\left(t_{n}\right)\right) \\
=\Phi_{A}\left(\frac{\Delta t}{2} ; \Phi_{B}\left(\frac{\Delta t}{2} ; v\left(t_{n}, t_{n}\right)\right)\right)-\Phi_{C}\left(\frac{\Delta t}{2} ; v\left(t_{n}, t_{n}\right)\right) \\
\quad+\Phi_{C}\left(\frac{\Delta t}{2} ; v\left(t_{n}, t_{n}\right)\right)-\Phi_{C}\left(\frac{\Delta t}{2} ; u\left(t_{n}\right)\right)-\left(v\left(t_{n}, t_{n}\right)-u\left(t_{n}\right)\right) \\
=\left(\Phi_{A}\left(\frac{\Delta t}{2} ; \Phi_{B}\left(\frac{\Delta t}{2} ; v\left(t_{n}, t_{n}\right)\right)\right)-\Phi_{C}\left(\frac{\Delta t}{2} ; v\left(t_{n}, t_{n}\right)\right)\right) \\
\quad+\left(\Phi_{C}\left(\frac{\Delta t}{2} ; \cdot\right)-I\right) \circ v\left(t_{n}, t_{n}\right)-\left(\Phi_{C}\left(\frac{\Delta t}{2} ; \cdot\right)-I\right) \circ u\left(t_{n}\right) .
\end{aligned}
$$

First we find that

$$
\left\|\Phi_{A}\left(\frac{\Delta t}{2} ; \Phi_{B}\left(\frac{\Delta t}{2} ; v\left(t_{n}, t_{n}\right)\right)\right)-\Phi_{C}\left(\frac{\Delta t}{2} ; v\left(t_{n}, t_{n}\right)\right)\right\|_{H^{s-9}} \leq K_{\alpha} \Delta t^{2}
$$

by using (41) (with $v\left(t_{n}, t_{n}\right)$ as initial data).

Introduce the function $V=V(x, t)$ satisfying

$$
V_{t}=V V_{x}-V_{x x x},\left.\quad V\right|_{t=t_{n}}=v\left(t_{n}, t_{n}\right)
$$

Then the very last line of (477) can be written as

$$
\begin{aligned}
& \left(\Phi_{C}\left(\frac{\Delta t}{2} ; \cdot\right)-I\right) \circ v\left(t_{n}, t_{n}\right)-\left(\Phi_{C}\left(\frac{\Delta t}{2} ; \cdot\right)-I\right) \circ u\left(t_{n}\right) \\
& \quad=\int_{t_{n}}^{t_{n+1 / 2}}\left(V_{t}(\sigma)-u_{t}(\sigma)\right) d \sigma \\
& \quad=\int_{t_{n}}^{t_{n+1 / 2}}\left(\frac{1}{2}\left(V(\sigma)^{2}\right)_{x}-\frac{1}{2}\left(u(\sigma)^{2}\right)_{x}-V_{x x x}(\sigma)+u_{x x x}(\sigma)\right) d \sigma \\
& =\int_{t_{n}}^{t_{n+1 / 2}}\left(\frac{1}{2}((V(\sigma)+u(\sigma))(V(\sigma)-u(\sigma)))_{x}-(V(\sigma)-u(\sigma))_{x x x}\right) d \sigma .
\end{aligned}
$$


Taking the $H^{s-9}$ norm we find

$$
\begin{aligned}
& \left\|\left(\Phi_{C}\left(\frac{\Delta t}{2} ; \cdot\right)-I\right) \circ v\left(t_{n}, t_{n}\right)-\left(\Phi_{C}\left(\frac{\Delta t}{2} ; \cdot\right)-I\right) \circ u\left(t_{n}\right)\right\|_{H^{s-9}} \\
& \leq \int_{t_{n}}^{t_{n+1 / 2}}\left(\frac{1}{2}\|(V(\sigma)+u(\sigma))(V(\sigma)-u(\sigma))\|_{H^{s-8}}+\|V(\sigma)-u(\sigma)\|_{H^{s-6}}\right) d \sigma \\
& \leq \int_{t_{n}}^{t_{n+1 / 2}}\left(K\left(\|V(\sigma)\|_{H^{s-8}}+\|u(\sigma)\|_{H^{s-8}}\right)\|V(\sigma)-u(\sigma)\|_{H^{s-8}}\right. \\
& \left.\quad+\|V(\sigma)-u(\sigma)\|_{H^{s-6}}\right) d \sigma \\
& \leq K_{\alpha} \int_{t_{n}}^{t_{n+1 / 2}}\|V(\sigma)-u(\sigma)\|_{H^{s-6}} d \sigma .
\end{aligned}
$$

By the $H^{k}$ stability of the KdV equation,

$$
\|V(\sigma)-u(\sigma)\|_{H^{s-6}} \leq K\left\|v\left(t_{n}, t_{n}\right)-u\left(t_{n}\right)\right\|_{H^{s-6}} \leq K_{\alpha} \Delta t,
$$

since by the arguments of Section 2.3 we have the estimate $\left\|w\left(t_{n}\right)\right\|_{H^{s-6}} \leq$ $\left\|w\left(t_{n}\right)\right\|_{H^{s-3}} \leq K_{\alpha} \Delta t$. Therefore

$$
\left\|\left(\Phi_{C}\left(\frac{\Delta t}{2} ; \cdot\right)-I\right) \circ v\left(t_{n}, t_{n}\right)-\left(\Phi_{C}\left(\frac{\Delta t}{2} ; \cdot\right)-I\right) \circ u\left(t_{n}\right)\right\|_{H^{s-9}} \leq K_{\alpha} \Delta t^{2} .
$$

Thus we have shown that

$$
\left\|w\left(t_{n+1 / 2}\right)-w\left(t_{n}\right)\right\|_{H^{s-9}} \leq K_{\alpha} \Delta t^{2} .
$$

The other terms on the right-hand side of (45) can be estimated in the same manner, using the expressions (46). Thus we conclude that (cf. (44))

$$
\|w(t)\|_{H^{s-9}} \leq K_{\alpha} \Delta t^{2} .
$$

If $t, \tau \in\left[t_{n}, t_{n+1 / 2}\right]$, we have $\|v(t, \tau)\|_{H^{\hat{k}}}=\|v(t, t)\|_{H^{\hat{k}}}$, and if $t, \tau \in\left[t_{n+1 / 2}, t_{n+1}\right]$, then an estimate analogous to (16) shows that

$$
\left|\|v(t, \tau)\|_{H^{\hat{k}}}-\|v(t, t)\|_{H^{\hat{k}}}\right| \leq K_{\alpha}|t-\tau| .
$$

The rest of the argument follows the procedure for the Godunov splitting. Now $s-9 \geq \hat{k}$ is the same as $s \geq 17$. Assuming this, we get

$$
\begin{aligned}
\|v(t, \tau)\|_{H^{\hat{k}}} & \leq\|v(t, t)\|_{H^{\hat{k}}}+\left|\|v(t, \tau)\|_{H^{\hat{k}}}-\|v(t, t)\|_{H^{\hat{k}}}\right| \\
& \leq K+K_{\alpha} \Delta t^{2}+K_{\alpha} \Delta t .
\end{aligned}
$$

Choosing $\alpha$ such that $K \leq \alpha / 4$, and then $\Delta t$ such that $K_{\alpha} \Delta t(\Delta t+1) \leq \alpha / 4$ implies that $\|v(t, \tau)\|_{H^{\hat{k}}} \leq \alpha / 2$. Hence by the bootstrap lemma and (44), the following holds:

Theorem 3.5. Fix $T>0$. Let $u_{0} \in H^{s}$ for some $s \geq 17$. Then for $\Delta t$ sufficiently small we have

$$
\|v(t, t)-u(t)\|_{H^{s-9}} \leq K \Delta t^{2}, \quad t \in[0, T],
$$

where the constant $K$ depends on $u_{0}, s$ and $T$ only. 
Remark 3.6. The result for Strang splitting is suboptimal. Possibilities for improvements include the following:

(i) By replacing the Sobolev spaces $H^{s}$ by Bourgain spaces $X^{s, b}$ one may improve the regularity hypothesis.

(ii) After the submission of this article, C. Lubich observed (private communication) that by using a different approach, one could reduce the order of the Sobolev space from 17 to 9 essentially by using the nonlinear variation of parameters formula and then using the midpoint rule to approximate the resulting error integrals.

\section{REFERENCES}

[1] A. Ambrosetti and G. Prodi. A Primer of Nonlinear Analysis. Cambridge Univ. Press, Cambridge, 1995. MR 1336591 (96a:58019)

[2] J. L. Bona and R. Smith. The initial-value problem for the Korteweg-de Vries equation. Philos. Trans. Roy. Soc. London Ser. A 278:555-601 (1975). MR0385355 (52:6219)

[3] H. Holden, K. H. Karlsen, and N. H. Risebro. Operator splitting methods for generalized Korteweg-de Vries equations. J. Comput. Phys. 153:203-222 (1999). MR1703652 (2001c:65101)

[4] H. Holden, K. H. Karlsen, K.-A. Lie, and N. H. Risebro. Splitting for Partial Differential Equations with Rough Solutions. European Math. Soc. Publishing House, Zürich, 2010.

[5] C. E. Kenig, G. Ponce, and L. Vega. Wellposedness of the initial value problem for the Korteweg-de Vries equation. J. Amer. Math. Soc. 4:323-347 (1991). MR.1086966 (92c:35106)

[6] F. Linares and G. Ponce. Introduction to Nonlinear Dispersive Equations. Springer, 2009. MR2492151

[7] C. Lubich. On splitting methods for Schrödinger-Poisson and cubic nonlinear Schrödinger equations. Math. Comp. 77:2141-2153 (2008). MR2429878(2009d:65114)

[8] T. Tao. Nonlinear Dispersive Equations. Local and Global Analysis. Amer. Math. Soc., Providence, RI, 2006. MR2233925 (2008i:35211)

[9] F. Tappert. Numerical solutions of the Korteweg-de Vries equation and its generalizations by the split-step Fourier method. In: (A. C. Newell, editor) Nonlinear Wave Motion, Amer. Math. Soc., 1974, pp. 215-216.

Department of Mathematical Sciences, Norwegian University of Science and Technology, NO-7491 Trondheim, Norway and Centre of Mathematics for Applications, University of Oslo, P.O. Box 1053, Blindern, NO-0316 Oslo, Norway

E-mail address: holden@math.ntnu.no

$U R L$ : www.math.ntnu.no/ holden

Centre of Mathematics for Applications, University of Oslo, P.O. Box 1053, BlinDERN, NO-0316 OSLO, Norway

E-mail address: kennethk@math.uio.no

$U R L$ : www.math.uio.no/ ${ }^{\sim}$ kennethk

Centre of Mathematics for Applications, University of Oslo, P.O. Box 1053, BlinDERN, NO-0316 OSLO, NORWAY

E-mail address: nilshr@math.uio.no

$U R L$ : www.math.uio.no/ nilshr

Department of Mathematics, University of California at Los Angeles, Los Angeles, CALifornia 90095-1555

E-mail address: tao@math.ucla.edu

$U R L$ : www.math.ucla.edu/ tao 